UWO-TH-07/01

MCTP-07-04

\title{
Thermodynamics of the $\mathcal{N}=2^{*}$ strongly coupled plasma
}

\author{
Alex Buchel ${ }^{1,2}$, Stan Deakin ${ }^{1}$, Patrick Kerner ${ }^{1}$ and James T. Liu ${ }^{3}$ \\ ${ }^{1}$ Department of Applied Mathematics \\ University of Western Ontario \\ London, Ontario N6A 5B\%, Canada \\ ${ }^{2}$ Perimeter Institute for Theoretical Physics \\ Waterloo, Ontario N2J 2W9, Canada \\ ${ }^{3}$ Michigan Center for Theoretical Physics \\ Randall Laboratory of Physics, The University of Michigan \\ Ann Arbor, MI 48109-1040, USA
}

\begin{abstract}
Gauge/string duality is a potentially important framework for addressing the properties of the strongly coupled quark gluon plasma produced at RHIC. However, constructing an actual string theory dual to QCD has so far proven elusive. In this paper, we take a partial step towards exploring the QCD plasma by investigating the thermodynamics of a non-conformal system, namely the $\mathcal{N}=2^{*}$ theory, which is obtained as a mass deformation of the conformal $\mathcal{N}=4$ gauge theory. We find that at temperatures of order the mass scale, the thermodynamics of the mass deformed plasma is surprisingly close to that of the conformal gauge theory plasma. This suggests that many properties of the quark gluon plasma at RHIC may in fact be well described by even relatively simple models such as that of the conformal $\mathcal{N}=4$ plasma.
\end{abstract}

January 2007 


\section{Introduction}

The purpose of this paper is to explore properties of strongly coupled nonconformal gauge theory plasma within the framework of the gauge theory/string theory correspondence of Maldacena [1,2]. We are primarily motivated by recent attempts (see [3] and references therein) to describe the quark gluon plasma (QGP) produced at RHIC [4-7] from the dual holographic perspective. Unfortunately, we do not as yet have a controllable string theory dual to QCD. In fact, most applications of the Maldacena correspondence to RHIC physics are discussed in the context of conformal $\mathcal{N}=4$ supersymmetric $S U(N)$ Yang-Mills (SYM) theory in the 't Hooft (planar) limit and for large 't Hooft coupling. Remarkably, $\mathcal{N}=4 \mathrm{SYM}$ plasma [8-16] as a model for the RHIC QGP appears to be reasonably good [17]. We would like to stress, however, that such an agreement is rather paradoxical. One requires a strong 't Hooft coupling in order to have a controllable string theory dual, and the QGP does indeed appear to be strongly coupled. However, it is strongly coupled because it is produced at temperatures of order the QCD strong coupling scale, where, naively, conformal invariance is badly broken. So, why then does the conformal gauge theory plasma serve as the model for RHIC physics?

As a first step towards answering the latter question, we study the thermodynamics of the $\mathcal{N}=2^{*}$ (i.e. mass deformed $\mathcal{N}=4$ ) SYM plasma over a wide range of temperatures and for different mass deformations ${ }^{1}$. The expectation here is that the deformation mass scale would provide a model for the QCD strong coupling scale, and the thermodynamics of the mass deformed plasma at temperatures of order the mass scale would then serve as a more realistic model for the RHIC QGP.

The paper is organized as follows. In the next section we review the non-extremal $\mathcal{N}=2^{*}$ geometry constructed in [21], the holographic renormalization of this theory [24] and also recall the explicit expressions for the free energy density, energy density and the entropy density $[24,25]$. In section 3 we discuss the map between finite temperature $\mathcal{N}=2^{*}$ gauge theory parameters and the parameters of the dual non-extremal geometry $[26,21]$. In section 4 we review the high-temperature thermo-

\footnotetext{
${ }^{1}$ Although there have been previous attempts to study the thermodynamics of strongly coupled nonconformal four dimensional gauge theory plasmas, they have been limited to the high temperature regime, where the theory is almost conformal [18-22]. A notable difference is a recent study of thermodynamics of strongly coupled $\mathcal{N}=2$ gauge theory plasma with massive fundamental hypermultiplets [23].
} 
dynamics of the finite temperature PW flow [21,24,25]. After this, we turn towards a numerical investigation of the flow away from the high-temperature regime. We describe our numerical procedure in section 5, and present the results of the analysis in section 6. Finally, we conclude and also outline open problems in section 7 .

\section{Non-extremal $\mathcal{N}=2^{*}$ geometry}

The supergravity background dual to finite temperature $\mathcal{N}=2^{*}$ gauge theory [21] is a deformation of the original $A d S_{5} \times S^{5}$ geometry induced by a pair of scalars $\alpha$ and $\chi$ of the five-dimensional gauge supergravity. (At zero temperature, such a deformation was constructed by Pilch and Warner [27].) According to the general scenario of a holographic RG flow, the asymptotic boundary behavior of the supergravity scalars is related to the bosonic and fermionic mass parameters of the relevant operators inducing the RG flow in the boundary gauge theory. Based on such a relation, and the fact that $\alpha$ and $\chi$ have conformal dimensions two and one, respectively, we call the supergravity scalar $\alpha$ a bosonic deformation, and the supergravity scalar $\chi$ a fermionic deformation of the D3-brane geometry.

The action of the five-dimensional gauged supergravity including the scalars $\alpha$ and $\chi$ is given by

$$
\begin{aligned}
S & =\int_{\mathcal{M}_{5}} d \xi^{5} \sqrt{-g} \mathcal{L}_{5} \\
& =\frac{1}{4 \pi G_{5}} \int_{\mathcal{M}_{5}} d \xi^{5} \sqrt{-g}\left[\frac{1}{4} R-3(\partial \alpha)^{2}-(\partial \chi)^{2}-\mathcal{P}\right]
\end{aligned}
$$

where the potential ${ }^{2}$

$$
\mathcal{P}=\frac{1}{16}\left[\frac{1}{3}\left(\frac{\partial W}{\partial \alpha}\right)^{2}+\left(\frac{\partial W}{\partial \chi}\right)^{2}\right]-\frac{1}{3} W^{2}
$$

is a function of $\alpha$ and $\chi$, and is determined by the superpotential

$$
W=-e^{-2 \alpha}-\frac{1}{2} e^{4 \alpha} \cosh (2 \chi) .
$$

In our conventions, the five-dimensional Newton's constant is

$$
G_{5} \equiv \frac{G_{10}}{2^{5} \operatorname{vol}_{S^{5}}}=\frac{4 \pi}{N^{2}} .
$$

\footnotetext{
${ }^{2}$ We set the five-dimensional gauged supergravity coupling to one. This corresponds to setting the radius $L$ of the five-dimensional sphere in the undeformed metric to 2 .
} 
The action (2.1) yields the Einstein equations

$$
R_{\mu \nu}=12 \partial_{\mu} \alpha \partial_{\nu} \alpha+4 \partial_{\mu} \chi \partial_{\nu} \chi+\frac{4}{3} g_{\mu \nu} \mathcal{P}
$$

as well as the equations for the scalars

$$
\square \alpha=\frac{1}{6} \frac{\partial \mathcal{P}}{\partial \alpha}, \quad \square \chi=\frac{1}{2} \frac{\partial \mathcal{P}}{\partial \chi} .
$$

To construct a finite-temperature version of the Pilch-Warner flow, we choose an ansatz for the metric respecting rotational but not the Lorentzian invariance

$$
d s_{5}^{2}=-c_{1}^{2}(r) d t^{2}+c_{2}^{2}(r)\left(d x_{1}^{2}+d x_{2}^{2}+d x_{3}^{2}\right)+d r^{2}
$$

With this ansatz, the equations of motion for the background become

$$
\begin{aligned}
& \alpha^{\prime \prime}+\alpha^{\prime}\left(\ln c_{1} c_{2}^{3}\right)^{\prime}-\frac{1}{6} \frac{\partial \mathcal{P}}{\partial \alpha}=0, \\
& \chi^{\prime \prime}+\chi^{\prime}\left(\ln c_{1} c_{2}^{3}\right)^{\prime}-\frac{1}{2} \frac{\partial \mathcal{P}}{\partial \chi}=0, \\
& c_{1}^{\prime \prime}+c_{1}^{\prime}\left(\ln c_{2}^{3}\right)^{\prime}+\frac{4}{3} c_{1} \mathcal{P}=0, \\
& c_{2}^{\prime \prime}+c_{2}^{\prime}\left(\ln c_{1} c_{2}^{2}\right)^{\prime}+\frac{4}{3} c_{2} \mathcal{P}=0,
\end{aligned}
$$

where the prime denotes a derivative with respect to the radial coordinate $r$. In addition, there is a first-order constraint

$$
\left(\alpha^{\prime}\right)^{2}+\frac{1}{3}\left(\chi^{\prime}\right)^{2}-\frac{1}{3} \mathcal{P}-\frac{1}{2}\left(\ln c_{2}\right)^{\prime}\left(\ln c_{1} c_{2}\right)^{\prime}=0 .
$$

It was shown in [21] that any solution to (2.8) and (2.9) can be lifted to a full tendimensional solution of type IIb supergravity. This includes the metric, the three- and five-form fluxes, the dilaton and the axion. In particular, the ten-dimensional Einstein frame metric is given by Eq. (4.12) in [21].

For finite temperature flows, we find it convenient to introduce a new radial coordinate $x$ which spans the range from the horizon to the boundary in finite coordinate distance:

$$
1-x(r)=\frac{c_{1}}{c_{2}}, \quad x \in[0,1] .
$$

With this new coordinate, the black brane's horizon is at $x=1$, while the boundary of the asymptotically $A d S_{5}$ space-time is at $x=0^{3}$. The background equations of motion

\footnotetext{
${ }^{3}$ This $x$ coordinate is inappropriate for the extremal PW flow, since in that case $x$ is always vanishing. In this sense, backgrounds with and without horizons (or equivalently physics at finite temperature and zero temperature) may have distinctly different characteristics.
} 
(2.8) become

$$
\begin{aligned}
& c_{2}^{\prime \prime}+4 c_{2}\left(\alpha^{\prime}\right)^{2}-\frac{1}{x-1} c_{2}^{\prime}-\frac{5}{c_{2}}\left(c_{2}^{\prime}\right)^{2}+\frac{4}{3} c_{2}\left(\chi^{\prime}\right)^{2}=0, \\
& \alpha^{\prime \prime}+\frac{1}{x-1} \alpha^{\prime}-\frac{1}{12 \mathcal{P} c_{2}^{2}(x-1)}\left[(x-1)\left(6\left(\alpha^{\prime}\right)^{2}+2\left(\chi^{\prime}\right)^{2}\right) c_{2}^{2}\right. \\
& \left.-3 c_{2}^{\prime} c_{2}-6\left(c_{2}^{\prime}\right)^{2}(x-1)\right] \frac{\partial \mathcal{P}}{\partial \alpha}=0, \\
& \chi^{\prime \prime}+\frac{1}{x-1} \chi^{\prime}-\frac{1}{4 \mathcal{P} c_{2}^{2}(x-1)}\left[(x-1)\left(6\left(\alpha^{\prime}\right)^{2}+2\left(\chi^{\prime}\right)^{2}\right) c_{2}^{2}\right. \\
& \left.-3 c_{2}^{\prime} c_{2}-6\left(c_{2}^{\prime}\right)^{2}(x-1)\right] \frac{\partial \mathcal{P}}{\partial \chi}=0,
\end{aligned}
$$

where the prime now denotes a derivative with respect to $x$. We demand that a physical RG flow should correspond to a background geometry with a regular horizon. To ensure regularity, it is necessary to impose the following boundary conditions at the horizon:

$$
x \rightarrow 1_{-}: \quad\left\{\alpha(x), \chi(x), c_{2}(x)\right\} \rightarrow\left\{\delta_{1}, \delta_{2}, \delta_{3}\right\}
$$

where the $\delta_{i}$ are constants.

The boundary conditions at $x=0$ are determined from the requirement that the solution should approach the $A d S_{5}$ geometry as $x \rightarrow 0_{+}$:

$$
x \rightarrow 0_{+}: \quad\left\{\alpha(x), \chi(x), c_{2}(x)\right\} \rightarrow\left\{0,0, \propto x^{-1 / 4}\right\}
$$

The three supergravity parameters $\delta_{i}$ uniquely determine a non-singular RG flow in the dual gauge theory. As we review in section 3, they are unambiguously related to the three physical parameters in the gauge theory: the temperature $T$, and the bosonic and fermionic masses $m_{b}$ and $m_{f}$ of the $\mathcal{N}=2^{*}$ hypermultiplet components.

A general analytical solution of the system (2.11) with the boundary conditions (2.12), (2.13) is unknown. However, it is possible to find an analytical solution in the regime of high temperatures ${ }^{4}$ (see section 4 ). Notice that given (2.2) and (2.3), one can consistently truncate the supergravity system (2.11) to bosonic deformation only, i.e., by setting $\chi=0$ identically. On the other hand, it is inconsistent (beyond the linear approximation) to set bosonic deformation to zero, i.e., to set $\alpha=0$ identically while keeping the fermionic deformation $\chi \neq 0$.

\footnotetext{
${ }^{4}$ Non-extremal $A d S_{5}$ geometry is obtained as a trivial solution for vanishing bosonic and fermionic deformations: $\alpha=\chi=0$ identically.
} 
In the rest of this section we discuss the asymptotic singularity-free solution of (2.11) near the boundary $x \rightarrow 0_{+}$and near the horizon $x \rightarrow 1_{-}$, constraint by the boundary conditions (2.13) and (2.12), respectively. We justify referring to the $\alpha$ and $\chi$ scalars as bosonic and fermionic deformations. We also explain the advantages of using the radial coordinate (2.10) in numerical integration. Finally, we recall holographic renormalization of the thermal PW flows.

In what follows we find it convenient to introduce

$$
\begin{aligned}
& c_{2} \equiv e^{A}, \quad A \equiv \ln \hat{\delta}_{3}-\frac{1}{4} \ln \left(2 x-x^{2}\right)+a(x), \\
& \rho \equiv e^{\alpha} .
\end{aligned}
$$

The form of $A(x)$ is chosen to extract the leading asymptotic behavior $c_{2} \sim x^{-1 / 4}$ from the metric function $c_{2}(x)$. In addition, the introduction of $\rho=e^{\alpha}$ is natural, as the bosonic scalar $\alpha$ enters exponentially in the superpotential (2.3). Imposing the boundary conditions (2.12) and (2.13), we see that the new warp factor $a(x)$ and scalar $\rho(x)$ satisfy the boundary conditions

$$
\begin{array}{ll}
x \rightarrow 0_{+}: & a(x) \rightarrow 0, \quad \rho(x) \rightarrow 1, \\
x \rightarrow 1_{-}: & a(x) \rightarrow \ln \frac{\delta_{3}}{\hat{\delta}_{3}}, \quad \rho(x) \rightarrow e^{\delta_{1}},
\end{array}
$$

where $\hat{\delta}_{3}$ is a new constant.

\subsection{Asymptotics of the thermal PW flow near the boundary}

The most general solution of (2.11) subject to the boundary conditions (2.13) and (2.15) may be expanded as a series around $x=0$ :

$$
\begin{aligned}
\rho= & +x^{1 / 2}\left(\rho_{10}+\rho_{11} \ln x\right)+x\left(\rho_{20}+\rho_{21} \ln x+\rho_{22} \ln ^{2} x\right) \\
& +\cdots+x^{k / 2}\left(\sum_{i=1}^{k} \rho_{k i} \ln ^{i} x\right)+\cdots, \\
\chi= & \chi_{0} x^{1 / 4}\left[1+x^{1 / 2}\left(\chi_{10}+\chi_{11} \ln x\right)+x\left(\chi_{20}+\chi_{21} \ln x+\chi_{22} \ln ^{2} x\right)\right. \\
& \left.+\cdots+x^{k / 2}\left(\sum_{i=1}^{k} \chi_{k i} \ln ^{i} x\right)+\cdots\right],
\end{aligned}
$$




$$
\begin{aligned}
a= & x^{1 / 2}\left(\alpha_{10}+\alpha_{11} \ln x\right)+x\left(\alpha_{20}+\alpha_{21} \ln x+\alpha_{22} \ln ^{2} x\right) \\
& +\cdots+x^{k / 2}\left(\sum_{i=1}^{k} \alpha_{k i} \ln ^{i} x\right)+\cdots .
\end{aligned}
$$

This solution is characterized by five independent parameters

$$
\left\{\ln \hat{\delta}_{3} ; \rho_{11}, \rho_{10} ; \chi_{0}, \chi_{10}\right\},
$$

where we have included $\hat{\delta}_{3}$ from (2.14). The remaining series coefficients are completely determined from the above parameters.

For the holographic renormalization (see subsection 2.3) we will need the coefficients of the first two subleading terms in (2.16)-(2.18). Explicitly, these are given by

$$
\begin{aligned}
& \rho_{22}=\frac{3}{2} \rho_{11}^{2} \\
& \rho_{21}=3 \rho_{10} \rho_{11}-8 \rho_{11}^{2}+\frac{26}{9} \chi_{0}^{2} \rho_{11} \\
& \rho_{20}=24 \rho_{11}^{2}+\frac{3}{2} \rho_{10}^{2}-8 \rho_{10} \rho_{11}+\frac{26}{9} \chi_{0}^{2} \rho_{10}-\frac{104}{9} \chi_{0}^{2} \rho_{11}+\frac{1}{3} \chi_{0}^{4}, \\
& \chi_{11}=\frac{1}{3} \chi_{0}^{2}, \\
& \chi_{22}=\frac{9}{2} \rho_{11}^{2}, \\
& \chi_{21}=2 \chi_{0}^{2} \rho_{11}+9 \rho_{10} \rho_{11}+\frac{13}{36} \chi_{0}^{4}-\frac{51}{2} \rho_{11}^{2}, \\
& \chi_{20}=\frac{1}{8}-6 \chi_{0} \rho_{11}+\frac{9}{2} \rho_{10}^{2}+\frac{243}{4} \rho_{11}^{2}-\frac{51}{2} \rho_{11} \rho_{10}+2 \chi_{0}^{2} \rho_{10}+\frac{13}{12} \chi_{0}^{2} \chi_{10}-\frac{131}{120} \chi_{0}^{4}, \\
& a_{11}=0, \\
& a_{10}=-\frac{1}{9} \chi_{0}^{2}, \\
& a_{22}=-\frac{1}{2} \rho_{11}^{2}, \\
& a_{21}=-\rho_{10} \rho_{11}-\frac{1}{2} \rho_{11}^{2}-\frac{1}{12} \chi_{0}^{4}, \\
& a_{20}=-\frac{1}{2} \rho_{10} \rho_{11}-\frac{1}{4} \chi_{0}^{2} \chi_{10}-\frac{3}{4} \rho_{11}^{2}+\frac{13}{648} \chi_{0}^{4}-\frac{1}{2} \rho_{10}^{2} .
\end{aligned}
$$

As indicated above, the non-extremal $A d S_{5}$ geometry is obtained by setting $\alpha \equiv 0$, $\chi \equiv 0$, which in asymptotic expansions (2.20)-(2.24) corresponds to taking $\rho_{11}=$ $\rho_{10}=\chi_{0}=0$. This leads to

$$
d s_{5}^{2}=(2 \pi T)^{2}\left(2 x-x^{2}\right)^{-1 / 2}\left(-(1-x)^{2} d t^{2}+d x_{1}^{2}+d x_{2}^{2}+d x_{3}^{2}\right)+\frac{d x^{2}}{2 x-x^{2}},
$$


where the temperature $T$ is given by

$$
2 \pi T=\hat{\delta}_{3}
$$

Matching with asymptotic extremal $A d S_{5}$ geometry with a standard Poincare patch radial coordinate $R$ (and with our conventional choice for the radius of curvature $L=2$ )

$$
d s_{5}^{2}=\frac{R^{2}}{4}\left(-d t^{2}+d x_{1}^{2}+d x_{2}^{2}+d x_{3}^{2}\right)+4 \frac{d R^{2}}{R^{2}},
$$

we identify near the boundary

$$
x \propto R^{-4}, \quad x \rightarrow 0_{+} .
$$

Given (2.28) and the asymptotic expansions (2.16)-(2.18), we identify the conformal weight two supergravity scalar $\rho(x)$ as dual to turning on mass for the bosonic components of the $\mathcal{N}=2^{*}$ hypermultiplet. Parameters $\rho_{11}$ and $\rho_{10}$ are coefficients of its non-normalizable and normalizable modes, correspondingly. Similarly, the conformal weight one supergravity scalar $\chi(x)$ can be identified as dual to turning on mass for the fermionic components of the $\mathcal{N}=2^{*}$ hypermultiplet. Parameters $\chi_{0}$ and $\chi_{10}$ are coefficients of its non-normalizable and normalizable modes, correspondingly. We discuss the precise relation of $\rho_{11}$ and $\chi_{0}$ to the $\mathcal{N}=2^{*}$ gauge theory bosonic and fermionic masses in section 3. Here, we would simply like to emphasize the following well-known fact [29]: given $\left\{\hat{\delta}, \rho_{11}, \chi_{0}\right\}$, the coefficients of the normalizable modes of the supergravity scalars $\rho(x)$ and $\chi(x)$, namely $\left\{\rho_{10}, \chi_{10}\right\}$, are uniquely fixed by requiring that the resulting supergravity RG flow is nonsingular in the bulk. This statement is simply the supergravity dual to a gauge theory lore: for a fixed temperature and mass parameters, the bosonic and fermionic condensates are determined uniquely ${ }^{5}$. Thus, for nonsingular finite temperature Pilch-Warner flows, we must have

$$
\rho_{10}=\rho_{10}\left(\hat{\delta}_{3}, \rho_{11}, \chi_{0}\right), \quad \chi_{10}=\chi_{10}\left(\hat{\delta}_{3}, \rho_{11}, \chi_{0}\right)
$$

\subsection{Asymptotics of the thermal PW flow near the horizon}

We now consider the behavior of the solution near the horizon, $x=1$. Notice that the equations (2.11) are invariant under the transformation

$$
(1-x) \Leftrightarrow-(1-x)
$$

\footnotetext{
${ }^{5}$ Strictly speaking this is true in the absence of moduli. However, we do not expect moduli at finite temperature, and thus unbroken supersymmetry, on the gauge theory side.
} 
It is the straightforward to verify that boundary conditions (2.12), i.e. the finiteness of $\{a(x), \rho(x), \chi(x)\}$ near the horizon, implies that $\{a(x), \rho(x), \chi(x)\}$ are even functions of $(1-x)$ for $|1-x| \ll 1$. It is this fact that justifies our choice of the radial coordinate $x$ in (2.10). Indeed, keeping fixed $\left\{\hat{\delta}_{3}, \rho_{11}, \chi_{0}\right\}$, for generic values of $\left\{\rho_{10}, \chi_{10}\right\}$, the functions $\{a(x), \rho(x), \chi(x)\}$ would diverge at the horizon. Thus, fixing coefficients of the normalizable modes $\left\{\rho_{10}, \chi_{10}\right\}$ from the requirement of nonsingularity of the holographic RG flow is equivalent to imposing Neumann boundary conditions on $\{a(x), \rho(x), \chi(x)\}$ at the horizon, i.e. as $x \rightarrow 1_{-}$

$$
\lim _{x \rightarrow 1_{-}} \rho^{\prime}=\lim _{x \rightarrow 1_{-}} \chi^{\prime}=\lim _{x \rightarrow 1_{-}} a^{\prime}=0 .
$$

The boundary condition (2.31) would determine bosonic and fermionic condensate dependence on the hypermultiplet masses and the temperature (2.29).

\subsection{Holographic thermodynamics of the finite temperature $\mathrm{PW}$ flow}

We use the method of holographic renormalization in order to examine the thermodynamics of the $\mathcal{N}=2^{*} \mathrm{RG}$ flow. Holographic renormalization of this flow was explained in [24], where it was investigated using the original radial coordinate $r$ of the metric ansatz (2.7). In particular, parameterizing

$$
c_{1}(r)=e^{A(r)+B(r)}, \quad c_{2}(r)=e^{A(r)},
$$

the results of [24] indicate that the entropy density $s$, the energy density $\mathcal{E}$, and the free energy density $\mathcal{F}$ are given by

$$
\begin{aligned}
& s=\frac{1}{4 G_{5}} \lim _{r \rightarrow r_{\text {horizon }}} e^{3 A}, \\
& \mathcal{E}=\frac{1}{8 \pi G_{5}} \lim _{r \rightarrow \infty}\left[-3 e^{4 A+B} A^{\prime}+2 e^{4 A+B}\left\{\alpha_{1}+\alpha_{3} \alpha+\alpha_{4} \chi^{2}+\alpha_{5} \alpha^{2}\right.\right. \\
& \left.\left.+\alpha_{6} \alpha \chi^{2}+\alpha_{8} \frac{\alpha^{2}}{\ln \epsilon}+\ln \epsilon \alpha_{10} \chi^{4}+\alpha_{11} \chi^{4}\right\}\right] \text {, } \\
& \mathcal{F}=\mathcal{E}-s T=\mathcal{E}-\frac{1}{8 \pi G_{5}} \lim _{r \rightarrow \infty}\left[e^{4 A+B} B^{\prime}\right],
\end{aligned}
$$

where

$$
\epsilon \equiv \sqrt{-g_{t t}}=e^{A+B}
$$


and the boundary counterterm coefficients $\alpha_{i}$ take the values

$$
\begin{aligned}
& \alpha_{1}=\frac{3}{4}, \quad \alpha_{2}=\frac{1}{4}, \\
& \alpha_{7}=0,
\end{aligned}
$$

Using the asymptotic expansions (2.16)-(2.18), and changing the radial coordinate in (2.33) following (2.10), we find

$$
\begin{gathered}
\mathcal{F}=-\frac{\hat{\delta}_{3}^{4}}{32 \pi G_{5}}\left(1+\rho_{11}^{2}\left(24-96 \ln \hat{\delta}_{3}+24 \ln 2\right)-24 \rho_{10} \rho_{11}+2 \chi_{0}^{2} \chi_{10}\right. \\
\left.+\chi_{0}^{4}\left(\frac{4}{9}-\frac{2}{3} \ln 2+\frac{8}{3} \ln \hat{\delta}_{3}\right)\right) \\
\mathcal{E}=\mathcal{F}-\frac{1}{8 \pi G_{5}} \hat{\delta}_{3}^{4}, \quad s T=\frac{1}{8 \pi G_{5}} \hat{\delta}_{3}^{4} .
\end{gathered}
$$

Finally, the entropy density is given by

$$
s=\frac{\hat{\delta}_{3}^{3} e^{3 a_{h}}}{4 G_{5}}, \quad a_{h}=\lim _{x \rightarrow 1_{-}} a(x),
$$

and thus we extract the temperature from $(2.37)$

$$
T=\frac{\hat{\delta}_{3}}{2 \pi} e^{-3 a_{h}}
$$

Of course, the same value of the Hawking temperature (2.39) can be extracted from the near-horizon geometry, provided one recalls that $s T$ is a renormalization group flow invariant in the supergravity black brane geometries without a chemical potential [30]. Indeed, from the relation between components of the Ricci tensor

$$
R_{x_{1}}^{x_{1}}=R_{t}^{t}
$$

we have a constraint ${ }^{6}$

$$
c_{2}^{4}\left(\frac{c_{1}}{c_{2}}\right)^{\prime}=\text { constant } .
$$

Evaluating the LHS of (2.41) near the horizon, we have

$$
\lim _{r \rightarrow r_{\text {horizon }}} c_{2}^{4}\left(\frac{c_{1}}{c_{2}}\right)^{\prime}=8 \pi G_{5} s T .
$$

\footnotetext{
${ }^{6}$ Eq. (2.41) can also be directly derived from (2.8).
} 
Changing to the radial coordinate $x$ in (2.10) and using the asymptotic expansions (2.16)-(2.18), the LHS of (2.41) near the boundary takes form ${ }^{7}$

$$
\lim _{r \rightarrow \infty} c_{2}^{4}\left(\frac{c_{1}}{c_{2}}\right)^{\prime}=\lim _{x \rightarrow 0_{+}}\left(-\frac{d x}{d r}\right) c_{2}^{4}=\hat{\delta}_{3}^{4} .
$$

Comparing (2.42) with (2.43) leads to the value of $s T$ presented in (2.37). It is precisely the holographic RG invariance of the combination $s T$ that guarantees the basic thermodynamic relation

$$
\mathcal{F}=\mathcal{E}-s T .
$$

Relation (2.44) was verified explicitly for the $\mathcal{N}=2^{*}$ thermal $R G$ flow in [21]; the same relation holds in other thermal gauge/gravity duals, for example in the thermal cascading RG flow [22].

\section{Gauge theory versus supergravity parameters}

The relation between $N=2^{*}$ gauge theory and the supergravity parameters of the (thermal) Pilch-Warner flow was established in $[26,21]$. For completeness, we review the main points here. We begin with the gauge theory, then move to the supersymmetric PW flow [26], and finally discuss the gauge/gravity parameter identification at finite temperature.

\section{$3.1 \quad N=2^{*}$ gauge theory}

In the language of four-dimensional $\mathcal{N}=1$ supersymmetry, the mass deformed $\mathcal{N}=4$ $S U(N)$ Yang-Mills theory $\left(\mathcal{N}=2^{*}\right)$ in $\mathbb{R}^{3,1}$ consists of a vector multiplet $V$, an adjoint chiral superfield $\Phi$ related by $\mathcal{N}=2$ supersymmetry to the gauge field, and two additional adjoint chiral multiplets $Q$ and $\tilde{Q}$ which form an $\mathcal{N}=2$ hypermultiplet. In addition to the usual gauge-invariant kinetic terms for these fields ${ }^{8}$, the theory has additional interactions and a hypermultiplet mass term given by the superpotential

$$
W=\frac{2 \sqrt{2}}{g_{Y M}^{2}} \operatorname{Tr}([Q, \tilde{Q}] \Phi)+\frac{m}{g_{Y M}^{2}}\left(\operatorname{Tr} Q^{2}+\operatorname{Tr} \tilde{Q}^{2}\right) .
$$

\footnotetext{
${ }^{7}$ In principle, one does not have to take a limit in (2.43) since this expression is a constant.

${ }^{8}$ The classical Kähler potential is normalized according to $\left(2 / g_{Y M}^{2}\right) \operatorname{Tr}[\bar{\Phi} \Phi+\bar{Q} Q+\overline{\tilde{Q}} \tilde{Q}]$.
} 
When $m=0$, the gauge theory is superconformal with $g_{Y M}$ characterizing an exactly marginal deformation. The theory has a classical $3(N-1)$ complex dimensional moduli space, which is protected by supersymmetry against (non)-perturbative quantum corrections.

When $m \neq 0$, the $\mathcal{N}=4$ supersymmetry is softly broken to $\mathcal{N}=2$. This mass deformation lifts the $\{Q, \tilde{Q}\}$ hypermultiplet moduli directions, leaving the $(N-1)$ complex dimensional Coulomb branch of the $\mathcal{N}=2 S U(N)$ Yang-Mills theory, parameterized by expectation values of the adjoint scalar

$$
\Phi=\operatorname{diag}\left(a_{1}, a_{2}, \cdots, a_{N}\right), \quad \sum_{i} a_{i}=0
$$

in the Cartan subalgebra of the gauge group. For generic values of the moduli $a_{i}$, the gauge symmetry is broken to that of the Cartan subalgebra $U(1)^{N-1}$, up to the permutation of individual $U(1)$ factors. Additionally, the superpotential (3.1) induces the $\mathrm{RG}$ flow of the gauge coupling. While from the gauge theory perspective it is straightforward to study this $\mathcal{N}=2^{*}$ theory at any point on the Coulomb branch [28], the PW supergravity flow [27] corresponds to a particular Coulomb branch vacuum. More specifically, matching the probe computation in gauge theory and the dual PW supergravity flow, it was argued in [26] that the appropriate Coulomb branch vacuum corresponds to a linear distribution of the vevs (3.2) as

$$
a_{i} \in\left[-a_{0}, a_{0}\right], \quad a_{0}^{2}=\frac{m^{2} g_{Y M}^{2} N}{\pi},
$$

with (continuous in the large $N$ limit) linear number density

$$
\rho(a)=\frac{2}{m^{2} g_{Y M}^{2}} \sqrt{a_{0}^{2}-a^{2}}, \quad \int_{-a_{0}}^{a_{0}} d a \rho(a)=N .
$$

Unfortunately, the extension of the $N=2^{*}$ gauge/gravity correspondence of $[27,26,31]$ for vacua other than (3.4) is not known.

In $[26,31]$ the dynamics of the gauge theory on the D3 brane probe in the PW background was studied in detail. It was shown in [26] that the probe has a one complex dimensional moduli space, with bulk induced metric precisely equal to the metric on the appropriate one complex dimensional submanifold of the $S U(N+1) \mathcal{N}=2^{*}$ DonagiWitten theory Coulomb branch. This one dimensional submanifold is parameterized by the expectation value $u$ of the $U(1)$ complex scalar on the Coulomb branch of the theory where $S U(N+1) \rightarrow U(1) \times S U(N)_{P W}$. Here the ${ }_{P W}$ subscript denotes 
that the $S U(N)$ factor is in the Pilch-Warner vacuum (3.4). Whenever $u$ coincides with any of the $a_{i}$ of the PW vacuum, the moduli space metric diverges, signaling the appearance of additional massless states. An identical divergence is observed [26,31] for the probe D3-brane at the enhancon singularity of the PW background. Away from the singularity locus, $u=a \in\left[-a_{0}, a_{0}\right]$, the gauge theory computation of the probe moduli space metric is one-loop exact. This is due to the suppression of instanton corrections in the large $N$ limit $[26,32]$ of $\mathcal{N}=2$ gauge theories.

Consider now $\mathcal{N}=2^{*}$ gauge theory at finite temperature $T$. Of course, finite temperature completely breaks supersymmetry. Thus, we can generalize the thermal $\mathcal{N}=2^{*}$ gauge theory by allowing for different (non-equal) masses $m_{b}$ and $m_{f}$ for the bosonic and fermionic components of the $\mathcal{N}=2^{*}$ hypermultiplet $\{Q, \tilde{Q}\}$ correspondingly. It is only when $m_{b}=m_{f}=m$, and at zero temperature $T=0$, that we have $\mathcal{N}=2$ supersymmetry. Since turning on mass terms for the bosonic or fermionic components of the hypermultiplet corresponds to deforming the $\mathcal{N}=4$ supersymmetric Yang-Mills Lagrangian by relevant operators of different classical dimension (a dimension two operator for bosonic and a dimension three operator for fermionic mass terms), such deformations will be encoded in different supergravity modes. As indicated in section 2, turning on bosonic/fermionic masses corresponds to turning on the five-dimensional supergravity scalars $\alpha / \chi$ correspondingly.

We would like to conclude this section with a simple observation. Turning on bosonic/fermionic masses for the hypermultiplet components sets a strong coupling scale $\Lambda \propto \max \left\{m_{b}, m_{f}\right\}$. In this case, we expect to find two qualitatively different phases of this gauge theory, depending on whether $T \gg \Lambda$ or $T \ll \Lambda$. In the former case we expect the thermodynamics to be qualitatively (and quantitatively in the limit $T / \Lambda \rightarrow \infty)$ similar to that of the $\mathcal{N}=4$ gauge theory plasma [33]. On the other hand, when $T \sim \Lambda$, and with $m_{f}=0$, we actually expect an instability in the system. Indeed $^{9}$, turning on only the supergravity scalar $\alpha$, i.e., setting $m_{b} \neq 0$ and $m_{f}=0$, corresponds to giving positive mass-squared to four out of six $\mathcal{N}=4$ scalars (these are the bosonic components of the $\mathcal{N}=2$ hypermultiplet). The remaining two $\mathcal{N}=4$ scalars at the same time obtain a negative mass-squared [27] — they are tachyons at zero temperature. At high enough temperature the thermal corrections would stabilize these tachyons. However, as we lower the temperature, we expect the re-emergence of these tachyons. As argued in [34], dynamical instabilities in thermal systems are

${ }^{9}$ We would like to thank Ofer Aharony for clarifying this. 
reflected in thermodynamic instabilities. Furthermore, it was argued in general (and demonstrated explicitly with a concrete example) [35] that thermodynamic instabilities are reflected to developing $c_{s}^{2}<0$, where $c_{s}$ is the speed of sound waves in the thermal gauge theory plasma. We discuss the supergravity realization of these phenomena in section 6 .

\subsection{Supersymmetric PW flow}

It is clear that the following conditions must hold in order to preserve $\mathcal{N}=2$ supersymmetry for the mass deformation of $\mathcal{N}=4 \mathrm{SYM}$ :

- the temperature must be zero: $T=0$;

- the masses for the bosonic and fermionic components of the $\mathcal{N}=2$ hypermultiplet $\{Q, \tilde{Q}\}$ must be the same: $m_{b}=m_{f}=m$.

The former condition corresponds to a restriction

$$
c_{1}(r)=c_{2}(r)
$$

ensuring Lorentz invariance of the metric. In this case, the supergravity RG flow cannot be parameterized by the radial coordinate $x$ introduced in (2.10). Instead, the supersymmetric flow is easiest to parameterize in terms of the fermionic scalar $\chi \in$ $[0,+\infty)$, where $\chi=0$ corresponds to the asymptotic $A d S_{5}$ boundary, and $\chi \rightarrow+\infty$ to the enhancon location in the bulk $[26,31]$. The supersymmetric Pilch-Warner solution is then given by [27]

$$
\begin{aligned}
e^{A} & =\frac{k \rho^{2}}{\sinh (2 \chi)}, \\
\rho^{6} & =\cosh (2 \chi)+\sinh ^{2}(2 \chi) \ln \frac{\sinh (\chi)}{\cosh (\chi)},
\end{aligned}
$$

where the single integration constant $k$ is related to the hypermultiplet mass $m$ according to $[26]$

$$
k=m L=2 m \text {. }
$$

In order to identify the thermal RG parameters $\left\{\rho_{11}, \chi_{0}\right\}$ with the gauge theory masses, we need the boundary asymptotics of the PW flow. This was computed in [21]. Introducing

$$
\hat{x} \equiv e^{-r / 2}
$$


we have

$$
\begin{aligned}
& \chi=k \hat{x}\left[1+k^{2} \hat{x}^{2}\left(\frac{1}{3}+\frac{4}{3} \ln (k \hat{x})\right)+k^{4} \hat{x}^{4}\left(-\frac{7}{90}+\frac{10}{3} \ln (k \hat{x})+\frac{20}{9} \ln ^{2}(k \hat{x})\right)\right. \\
& \left.+\mathcal{O}\left(k^{6} \hat{x}^{6} \ln ^{3}(k \hat{x})\right)\right] \\
& \rho=1+k^{2} \hat{x}^{2}\left(\frac{1}{3}+\frac{2}{3} \ln (k \hat{x})\right)+k^{4} \hat{x}^{4}\left(\frac{1}{18}+2 \ln (k \hat{x})+\frac{2}{3} \ln ^{2}(k \hat{x})\right)+\mathcal{O}\left(k^{6} \hat{x}^{6} \ln ^{3}(k \hat{x})\right), \\
& A=-\ln (2 \hat{x})-\frac{1}{3} k^{2} \hat{x}^{2}-k^{4} \hat{x}^{4}\left(\frac{2}{9}+\frac{10}{9} \ln (k \hat{x})+\frac{4}{9} \ln ^{2}(k \hat{x})\right)+\mathcal{O}\left(k^{6} \hat{x}^{6} \ln ^{3}(k \hat{x})\right) \text {. }
\end{aligned}
$$

\subsection{Thermal PW flow}

We now consider the thermal PW flow with $m_{b}=m_{f}=m$. Here we would like to identify the parameters $\rho_{11}$ and $\chi_{0}$ for such a flow in terms of the PW quantity $k$ (and thus, following (3.7), the hypermultiplet mass $m$ ) by comparing the boundary asymptotics $(2.16)-(2.18)$ with (3.9). In order to do so, we first establish the relation between the radial coordinate $x$ in $(2.16)-(2.18)$ and the radial coordinate $\hat{x}$ in (3.9). Matching the asymptotic warp factors $e^{A}$ for the zero temperature PW flow and the thermal PW flow as $x \rightarrow 0_{+}$, we find

$$
x \sim 8 \hat{\delta}_{3}^{4} \hat{x}^{4}, \quad x \rightarrow 0_{+} .
$$

Next, using (3.10) and matching the leading nontrivial asymptotics [order $x^{1 / 2} \ln x$ for $\rho(x)$ and order $x^{1 / 4}$ for $\left.\chi(x)\right]$ for both the thermal PW solution and the supersymmetric PW flow, we find

$$
\rho_{11}=\frac{2^{1 / 2} k^{2}}{24 \hat{\delta}_{3}^{2}}=\frac{\sqrt{2}}{24 \pi^{2}} e^{-6 a_{h}}\left(\frac{m}{T}\right)^{2}, \quad \chi_{0}=\frac{2^{1 / 4} k}{2 \hat{\delta}_{3}}=\frac{1}{2^{3 / 4} \pi} e^{-3 a_{h}}\left(\frac{m}{T}\right),
$$

where we used (2.39). We emphasize that the above matching procedure is welldefined - it was shown in [21] that finite temperature effects modify the asymptotic supersymmetric PW geometry at order $\mathcal{O}(x) \sim \mathcal{O}\left(\hat{x}^{4}\right)$, which is subleading compared to the order at which the matching $(3.11)$ is done.

Motivated by (3.11), we now propose that in the general case, i.e. for $m_{b} \neq m_{f}$, we may independently extract bosonic and fermionic masses according to

$$
\rho_{11}=\frac{\sqrt{2}}{24 \pi^{2}} e^{-6 a_{h}}\left(\frac{m_{b}}{T}\right)^{2}, \quad \chi_{0}=\frac{1}{2^{3 / 4} \pi} e^{-3 a_{h}}\left(\frac{m_{f}}{T}\right) .
$$

In section 4 we recall that such an identification leads to a consistent thermodynamics of the thermal PW flows $[24,25]$ at high temperatures. Furthermore, we later show in 
section 6 that this identification in fact leads to a consistent thermodynamics at any temperature.

In the rest of this subsection we derive some useful expressions for the free energy and the speed of sound waves. Specifically, we would like to express the free energy and the speed of sound in terms of the coefficients of the non-normalizable modes of the supergravity scalars $\rho(x), \chi(x)$. We also present the differential constraint on coefficients of the normalizable modes of these supergravity scalars following from the first law of thermodynamics.

Since we will constantly refer to the parameters $\rho_{11}$ and $\chi_{0}$ related to the bosonic and fermionic masses, we distinguish them from derived quantities (such as the subleading coefficients) by introducing the notation

$$
\xi \equiv \rho_{11}, \quad \eta \equiv \chi_{0} .
$$

Notice that because of (3.12), $\xi$ and $\eta$ are not independent, but are related through the ratio of bosonic and fermionic masses according to

$$
\eta^{2}=6 \frac{m_{f}^{2}}{m_{b}^{2}} \xi
$$

The nonsingularity of the thermal PW flow at the horizon (2.31) will determine the coefficients $\left\{\rho_{10}, \chi_{10}\right\}$ of the normalizable modes of the supergravity scalars $\{\rho(x), \chi(x)\}$ and also the value $a_{h}$ of the warp factor $a(x)$ at the horizon in terms of (3.13) and (3.14). When $m_{b} \neq 0$, we take

$$
\rho_{10}=\rho_{10}(\xi), \quad \chi_{10}=\chi_{10}(\xi), \quad a_{h}=a_{h}(\xi) .
$$

On the other hand, for $m_{b}=0$, we have instead

$$
\rho_{10}=\rho_{10}(\eta), \quad \chi_{10}=\chi_{10}(\eta), \quad a_{h}=a_{h}(\eta) .
$$

Notice that a given pair $\{\xi, \eta\}$ can be unambiguously related to bosonic and fermionic masses, measured with respect to temperature. Indeed, following (3.12)

$$
\left(\frac{m_{b}}{T}\right)^{2}=12 \sqrt{2} \pi^{2} e^{6 a_{h}} \xi, \quad\left(\frac{m_{f}}{T}\right)=2^{3 / 4} \pi e^{3 a_{h}} \eta
$$

Solving for $\hat{\delta}_{3}$ from (2.39) and expressing $G_{5}$ in gauge theory variables (2.4), we find for the free energy

$$
\begin{aligned}
\mathcal{F}= & -\frac{1}{8} \pi^{2} N^{2} T^{4} e^{12 a_{h}(\xi)}\left(1+24 \xi^{2} \ln \left(\xi^{2}\right)\left(1-\frac{m_{f}^{4}}{m_{b}^{4}}\right)+12 \xi \frac{m_{f}^{2}}{m_{b}^{2}} \chi_{10}(\xi)-24 \xi \rho_{10}(\xi)\right) \\
& +\mathcal{F}_{0},
\end{aligned}
$$


assuming $m_{b} \neq 0$, and

$$
\mathcal{F}=-\frac{1}{8} \pi^{2} N^{2} T^{4} e^{12 a_{h}(\eta)}\left(1-\frac{2}{3} \eta^{4} \ln \left(\eta^{4}\right)+2 \eta^{2} \chi_{10}(\eta)\right)+\mathcal{F}_{0},
$$

if $\xi=0$. In the above expressions, $\mathcal{F}_{0}$ does not depend on temperature. Such a constant arises because holographic renormalization generically ${ }^{10}$ leads to $\ln T$ dependence in the free energy, which requires the introduction of an arbitrary regularization scale $\mu$

$$
\ln T \rightarrow \ln \frac{T}{\mu}
$$

Changing this scale modifies $\mathcal{F}_{0}$, but otherwise has no effect on the thermodynamics. This phenomena has been encountered previously; it was discussed in perturbative gauge theory thermodynamics in [36], and in the context of gauge/string theory correspondence in $[37]^{11}$. Notice that in $(3.18)$ we wrote $\ln \left(\xi^{2}\right)$ instead of $2 \ln (\xi)$. The reason is that the former expression allows us to study thermodynamics for $\xi<0$, which according to $(3.12)$ we interpret as $m_{b}^{2}<0$. While introducing tachyonic masses for gauge theory scalars at zero temperature leads to instability, this is not the case at finite temperature. Here, the effective mass squared receives thermal corrections of order $T^{2}$, which might cure the zero temperature instability.

Besides the free energy, we can also evaluate the entropy density and the energy density

$$
s=\frac{1}{2} \pi^{2} N^{2} T^{3} e^{12 a_{h}}, \quad \mathcal{E}=\mathcal{F}+s T .
$$

In addition, from the defining equations (3.17), we can evaluate the quantities $d \xi / d T$ and $d \eta / d T$ :

$$
\begin{array}{ll}
\frac{d \xi}{d T}=-\frac{m_{b}^{2}}{6 \sqrt{2} \pi^{2} T^{3}} e^{-6 a_{h}(\xi)}\left(6 \xi \frac{d a_{h}(\xi)}{d \xi}+1\right)^{-1}, & m_{b} \neq 0 \\
\frac{d \eta}{d T}=-\frac{m_{f}}{2^{3 / 4} \pi T^{2}} e^{-3 a_{h}(\eta)}\left(3 \eta \frac{d a_{h}(\eta)}{d \eta}+1\right)^{-1}, & m_{b}=0
\end{array}
$$

Using (3.18) or (3.19), (3.21) and (3.22) the first law of thermodynamics,

$$
d \mathcal{F}=-s d T
$$

\footnotetext{
${ }^{10}$ Notice that this is not the case when $m_{b}=m_{f}$.

${ }^{11}$ It appears even in the high temperature thermodynamics of the thermal PW flow [25] (see also section 4).
} 
reduces to a differential constraint on the quantities ${ }^{12}$

$$
\begin{array}{ll}
\left\{\xi, \chi_{10}(\xi), \rho_{10}(\xi), a_{h}(\xi)\right\} & \text { for } m_{b} \neq 0 \\
\left\{\eta, \chi_{10}(\eta), \rho_{10}(\eta), a_{h}(\eta)\right\} & \text { for } m_{b}=0 .
\end{array}
$$

In particular, the first law in differential form reads

$$
0=4 \xi\left(1-\frac{m_{f}^{4}}{m_{b}^{4}}\right)+2\left(\rho_{10}(\xi)-\xi \frac{d \rho_{10}(\xi)}{d \xi}\right)-\frac{m_{f}^{2}}{m_{b}^{2}}\left(\chi_{10}(\xi)-\xi \frac{d \chi_{10}(\xi)}{d \xi}\right)+\frac{d a_{h}(\xi)}{d \xi}
$$

for $m_{b} \neq 0$, and

$$
0=\frac{4}{3} \eta^{3}+2 \eta \chi_{10}(\eta)-\eta^{2} \frac{d \chi_{10}(\eta)}{d \eta}-6 \frac{d a_{h}(\eta)}{d \eta}
$$

for $m_{b}=0$. Using either (3.25) or (3.26), we can evaluate the speed of sound entirely as a function of $\xi$ or $\eta$. Straightforward algebraic manipulations result in

$$
c_{s}^{2}=\frac{\partial P}{\partial \mathcal{E}}=-\frac{\partial \mathcal{F}}{\partial \mathcal{E}}=\frac{1+\Sigma}{3-\Sigma}
$$

where

$$
\begin{aligned}
\Sigma \equiv \Sigma(\xi)= & 24 \xi^{2}\left(\frac{m_{f}^{4}}{m_{b}^{4}}-1\right)+12 \xi\left(\xi \frac{d \rho_{10}(\xi)}{d \xi}-\rho_{10}(\xi)\right) \\
& +6 \xi \frac{m_{f}^{2}}{m_{b}^{2}}\left(\chi_{10}(\xi)-\xi \frac{d \chi_{10}(\xi)}{d \xi}\right), \quad m_{b} \neq 0 \\
\Sigma \equiv \Sigma(\eta)= & \frac{2}{3} \eta^{4}+\eta^{2} \chi_{10}(\eta)-\frac{1}{2} \eta^{3} \frac{d \chi_{10}(\eta)}{d \eta}, \quad m_{b}=0
\end{aligned}
$$

and where $P=-\mathcal{F}$ is the pressure.

In general, one may explore the PW thermodynamics in the entire phase plane of $m_{b}-m_{f}$. However, here we focus on two special cases:

- when $m_{f}=0$ (we call this the bosonic case) ${ }^{13}$ we have

$$
\begin{gathered}
\mathcal{F}_{\text {bosonic }}=-\frac{\pi^{2} N^{2} T^{4}}{8} e^{12 a_{h}(\xi)}\left(1+24 \xi^{2} \ln \left(\xi^{2}\right)-24 \xi \rho_{10}(\xi)\right)+\mathcal{F}_{0}, \\
\sum_{\text {bosonic }}(\xi)=-24 \xi^{2}-12 \xi \rho_{10}(\xi)+12 \xi^{2} \frac{d \rho_{10}(\xi)}{d \xi}
\end{gathered}
$$

\footnotetext{
${ }^{12}$ Notice that even though, for $\xi=0$, the free energy density (3.19) does not depend on $\rho_{10}$, one generically expects that $\rho_{10}=\rho_{10}(\eta)$.

${ }^{13}$ Recall that this corresponds to a consistent truncation of the supergravity
} 
and the first law of thermodynamics takes form

$$
0=4 \xi+2\left(\rho_{10}(\xi)-\xi \frac{d \rho_{10}(\xi)}{d \xi}\right)+\frac{d a_{h}(\xi)}{d \xi} ;
$$

- when $m_{f}=m_{b} \equiv m$ (we call this the supersymmetric case), or correspondingly from $(3.14)$

$$
\eta^{2}=6 \xi
$$

we have

$$
\begin{gathered}
\mathcal{F}_{\text {susy }}=-\frac{\pi^{2} N^{2} T^{4}}{8} e^{12 a_{h}(\xi)}\left(1-24 \xi \rho_{10}(\xi)+12 \xi \chi_{10}(\xi)\right)+\mathcal{F}_{0}, \\
\Sigma_{\text {susy }}(\xi)=12 \xi\left(\xi \frac{d \rho_{10}(\xi)}{d \xi}-\rho_{10}(\xi)\right)+6 \xi\left(\chi_{10}(\xi)-\xi \frac{d \chi_{10}(\xi)}{d \xi}\right),
\end{gathered}
$$

and the first law of thermodynamics takes form

$$
0=2\left(\rho_{10}(\xi)-\xi \frac{d \rho_{10}(\xi)}{d \xi}\right)-\left(\chi_{10}(\xi)-\xi \frac{d \chi_{10}(\xi)}{d \xi}\right)+\frac{d a_{h}(\xi)}{d \xi}
$$

Notice that in the supersymmetric case there is no $\ln \left(\xi^{2}\right)$ dependence in the holographic free energy (3.18). As a result one can unambiguously determine $\mathcal{F}_{0}$ directly from $(2.33)^{14}$. We find

$$
\mathcal{F}_{0} \equiv \mathcal{F}_{0}^{\text {susy }}=-\frac{\pi^{2} N^{2} T^{4}}{8} e^{12 a_{h}(\xi)} 40 \xi^{2}=-\frac{5 N^{2}}{288 \pi^{2}} m^{4} .
$$

\section{Thermodynamics of the high temperature Pilch-Warner flow}

We now recall that the differential equations (2.11) describing the finite temperature PW renormalization group flow admit a perturbative analytical solution at high temperature $[21,24,25]$. In this regime, the appropriate expansion parameters are

$$
\delta_{1} \propto\left(\frac{m_{b}}{T}\right)^{2} \ll 1, \quad \delta_{2} \propto \frac{m_{f}}{T} \ll 1 .
$$

In this case, the metric function $A(x)$ is given to first nontrivial order in $\delta_{1}, \delta_{2}$ by

$$
\begin{aligned}
& A(x)=\ln \delta_{3}-\frac{1}{4} \ln \left(2 x-x^{2}\right)+\delta_{1}^{2} A_{1}(x)+\delta_{2}^{2} A_{2}(x), \\
& \alpha(x)=\delta_{1} \alpha_{1}(x) \\
& \chi(x)=\delta_{2} \chi_{2}(x)
\end{aligned}
$$

\footnotetext{
${ }^{14}$ The latter holographic prescription was formulated in such a way that the holographic energy of the supersymmetric PW flow vanishes [24].
} 
where

$$
\begin{gathered}
\alpha_{1}=\left(2 x-x^{2}\right)_{2}^{1 / 2}{ }_{2} F_{1}\left(\frac{1}{2}, \frac{1}{2} ; 1 ;(1-x)^{2}\right), \\
\chi_{2}=\left(2 x-x^{2}\right)^{3 / 4}{ }_{2} F_{1}\left(\frac{3}{4}, \frac{3}{4} ; 1 ;(1-x)^{2}\right), \\
A_{1}=4 \int_{x}^{1} \frac{(z-1) d z}{\left(2 z-z^{2}\right)^{2}}\left(\gamma_{1}-\int_{z}^{1} d y\left(\frac{\partial \alpha_{1}}{\partial y}\right)^{2} \frac{\left(2 y-y^{2}\right)^{2}}{y-1}\right), \\
A_{2}=\frac{4}{3} \int_{x}^{1} \frac{(z-1) d z}{\left(2 z-z^{2}\right)^{2}}\left(\gamma_{2}-\int_{z}^{1} d y\left(\frac{\partial \chi_{2}}{\partial y}\right)^{2} \frac{\left(2 y-y^{2}\right)^{2}}{y-1}\right) .
\end{gathered}
$$

The constants $\gamma_{i}$ are fine-tuned to satisfy the boundary conditions, and are given by

$$
\gamma_{1}=\frac{8-\pi^{2}}{2 \pi^{2}}, \quad \gamma_{2}=\frac{8-3 \pi}{8 \pi} .
$$

Comparing (4.3), (4.4) and (2.16), (2.17) we find the relations

$$
\begin{array}{ll}
\rho_{11}=-\frac{\sqrt{2}}{\pi} \delta_{1}, & \rho_{10}=\frac{3 \sqrt{2} \ln 2}{\pi} \delta_{1}, \\
\chi_{0}=\frac{2^{1 / 4} \sqrt{\pi}}{\Gamma\left(\frac{3}{4}\right)^{2}} \delta_{2}, & \chi_{10}=-\frac{2^{1 / 2} \Gamma\left(\frac{3}{4}\right)^{4}}{\pi^{2}} .
\end{array}
$$

Also notice that

$$
\begin{aligned}
2 \pi T & =\delta_{3}\left(1+\delta_{1}^{2}\left(2-\left.\frac{d^{2} A_{1}}{d x^{2}}\right|_{x=1}\right)+\delta_{2}^{2}\left(\frac{1}{2}-\left.\frac{d^{2} A_{2}}{d x^{2}}\right|_{x=1}\right)\right) \\
& =\delta_{3}\left(1+\frac{16}{\pi^{2}} \delta_{1}^{2}+\frac{4}{3 \pi} \delta_{2}^{2}\right) .
\end{aligned}
$$

The parameters $\left\{\delta_{1}, \delta_{2}\right\}$ can be related to the $\left\{m_{b} / T, m_{f} / T\right\}$ parameters of the dual gauge theory via

$$
\begin{aligned}
& \delta_{1}=-\frac{1}{24 \pi}\left(\frac{m_{b}}{T}\right)^{2}, \\
& \delta_{2}=\frac{\left[\Gamma\left(\frac{3}{4}\right)\right]^{2}}{2 \pi^{3 / 2}} \frac{m_{f}}{T} .
\end{aligned}
$$

To leading order in mass deformation, the free energy (3.18) takes form $[24,25]$

$$
\mathcal{F}=-\frac{1}{8} \pi^{2} N^{2} T^{4}\left[1-\frac{192}{\pi^{2}} \ln (\pi T) \delta_{1}^{2}-\frac{8}{\pi} \delta_{2}^{2}\right] .
$$

One can further evaluate the entropy density of the non-extremal PW geometry,

$$
s=\frac{1}{2} \pi^{2} N^{2} T^{3}\left(1-\frac{48}{\pi^{2}} \delta_{1}^{2}-\frac{4}{\pi} \delta_{2}^{2}\right),
$$

and verify that the first law of thermodynamics (3.23) is satisfied. 


\section{$5 \quad$ Numerical procedure and results}

We are mainly interested in the thermodynamics of the PW flow away from the high temperature limit. In this case, since analytic solutions to the equations of motion (2.11) are unavailable, we resort to numerical integration. In this section we describe the numerical procedure and some of the consistency checks we have performed on the numerical results. Extracting the actual thermodynamics from these solutions will be taken up in the following section.

The numerical procedure is conceptually the same for both the bosonic and supersymmetric mass deformations of the non-extremal $A d S_{5}$ geometry. In practice, however, it is much easier to implement for the bosonic deformation

$$
m_{f}=0, \quad m_{b} \neq 0,
$$

and so we will describe the details of this first. We then outline the modifications required for handling the supersymmetric mass deformation

$$
m_{f}=m_{b} \equiv m
$$

and comment on thermal flows corresponding to generic mass deformations. Finally, we discuss the validity of the supergravity approximation.

\subsection{Bosonic deformation}

Simplicity of treating the bosonic mass deformation stems from the fact that thermal PW flows allow for a consistent truncation with the supergravity scalar $\chi(x)$ identically set to zero. The relevant equations can thus be obtained from (2.11) with

$$
\chi(x) \equiv 0, \quad \alpha \equiv \ln \rho, \quad \ln c_{2}(x) \equiv A(x) \equiv \ln \hat{\delta}_{3}-\frac{1}{4} \ln \left(2 x-x^{2}\right)+a(x) .
$$

The boundary conditions (which guarantee that the flow is singularity-free) are given by $(2.15)$ and $(2.31)$

$$
\begin{array}{lll}
x \rightarrow 0_{+} & a(x) \rightarrow 0, & \rho(x) \rightarrow 1 ; \\
x \rightarrow 1_{-} & a^{\prime}(x) \rightarrow 0, & \rho^{\prime}(x) \rightarrow 0 .
\end{array}
$$

Asymptotic expansions for $\{\rho(x), a(x)\}$ as $x \rightarrow 0_{+}$are given by (2.16), (2.18). The first couple terms are presented in $(2.20)-(2.24)$, where one has to set $\chi_{0}=0$ corresponding 
to $\chi(x) \equiv 0$, see (2.17). Notice that numerical integration of $\rho(x)$ and $a(x)$ from $x=0_{+}$is uniquely determined specifying the non-normalizable $\rho_{11}$ and normalizable $\rho_{10}$ mode coefficients. However, a generic choice of a pair $\left\{\rho_{11}, \rho_{10}\right\}$ would produce a solution that does not satisfy the horizon boundary condition (the $x \rightarrow 1_{-}$boundary condition in (5.4)). Solving Sturm-Liouville problem with boundary conditions (5.4) would determine

$$
\rho_{10} \equiv \rho_{10}\left(\rho_{11}\right) \quad \Longleftrightarrow \quad \rho_{10} \equiv \rho_{10}(\xi)
$$

where on the RHS we used conventions of subsection 3.3. For a flow satisfying (5.5) we can extract

$$
a_{h}(\xi)=\lim _{x \rightarrow 1_{-}} a(x)
$$

To summarize, each non-singular bosonic deformation flow would generate a triplet of numbers

$$
\left\{\xi, \rho_{10}(\xi), a_{h}(\xi)\right\}
$$

which can be used to evaluate the free energy $\mathcal{F}_{\text {bosonic }}(3.29)$, the speed of sound $c_{s, \text { bosonic }}^{2}$ (3.27) and (3.30), and verify (numerically) the first law of thermodynamics (3.31).

We use the following algorithm to generate triplets (5.7). First, we choose a mass deformation parameter $\rho_{11}=\xi$ which we keep fixed during the iteration procedure. The iteration starts by choosing a trial value of $\rho_{10}$. Given $\left\{\rho_{11}, \rho_{10}\right\}$ we integrate numerically $^{15}(2.11)$ (with $\left.(5.3)\right)$ from $x_{\text {initial }} \ll 1$ to $x_{\text {final }}\left(\right.$ such that $\left(1-x_{\text {final }}\right) \ll 1$ ) using the power series $(2.16),(2.17)$ to specify ${ }^{16}$

$$
\rho\left(x_{\text {initial }}\right), \quad \rho^{\prime}\left(x_{\text {initial }}\right), \quad a\left(x_{\text {initial }}\right), \quad a^{\prime}\left(x_{\text {initial }}\right) .
$$

For a generic value of $\rho_{10}$,

$$
\lim _{x \rightarrow 1_{-}} \rho(x) \rightarrow \pm \infty, \quad \lim _{x \rightarrow 1_{-}} \alpha(x) \rightarrow-\infty
$$

We find that for a given value $\rho_{11}=\xi$ there is (generically) a unique $\rho_{10}=\rho_{10}(\xi)$, such that $\rho(x)$ and $a(x)$ are finite as we go to the horizon, $x \rightarrow 1_{-}$, i.e.,

$$
\lim _{x \rightarrow 1_{-}} \frac{d \rho}{d x}\left(x ; \rho_{11}=\xi, \hat{\rho}_{10}=\rho_{10}(\xi)\right)=0
$$

\footnotetext{
${ }^{15}$ We used Mathematica for numerical integration as well as our own $C$ code based on fifth-order Runge-Kutta. Both procedures (up to controllable numerical errors) produced equivalent results.

${ }^{16}$ In practice we truncated the power series to $k=3$ terms (inclusive), and used $x_{\text {initial }}=10^{-9}$, $x_{\text {final }}=1-10^{-6}$.
} 


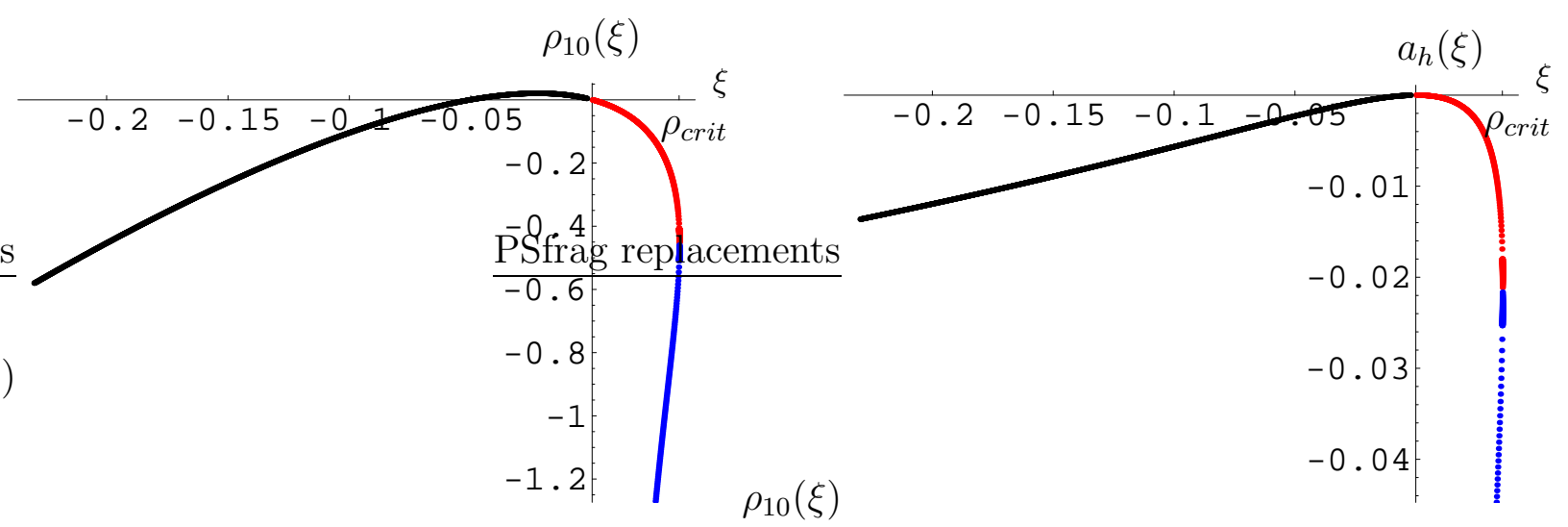

Figure 1: Altogether we obtained 3227 triplets $\left\{\xi, \rho_{10}(\xi), a_{h}(\xi)\right\}$. The left plot represents $\rho_{10}(\xi)$, and the right plot represents $a_{h}(\xi)$. The critical value of $\xi$ is at $\rho_{\text {crit }} \approx 0.03582(2)$. Red segments of the plots correspond to $\xi \in\left[0, \rho_{\text {crit }}\right)$ such that $\rho_{10}(0)=a_{h}(0)=0$. Blue segments of the plots do not pass through the origin and correspond to $0<\xi<\rho_{\text {crit }}$. Black segments of the plots correspond to $\xi<0$.

while

$$
\lim _{x \rightarrow 1_{-}} \frac{d \rho}{d x}\left(x ; \rho_{11}=\xi, \rho_{10} \neq \rho_{10}(\xi)\right)= \pm \infty .
$$

Now, the iterative algorithm is clear: we use Newton's shooting method of determining $\rho_{10}(\xi)$ for a fixed $\rho_{11}=\xi$.

Results of the numerical analysis are presented in Figs. 1-3. From Fig. 1 notice that for $\rho_{11}>\rho_{\text {crit }} \approx 0.03582$ there is no solution to Sturm-Liouville problem with boundary condition (5.4). Using (3.17) we find that corresponding to $\rho_{\text {crit }}$,

$$
\left.\frac{m_{b}}{T}\right|_{\text {crit }} \approx 2.29(9) \text {. }
$$

We find that for each value of $\xi \in\left(0, \rho_{\text {crit }}\right)$ there are two sets $\left\{\rho_{10}(\xi), a_{h}(\xi)\right\}$ which are represented by red/blue segments of the plots in Fig. 1. For each $\xi<0$ we find a single set $\left\{\rho_{10}(\xi), a_{h}(\xi)\right\}$ represented by black segments of the plots in Fig. 1 . In section 6 we show that red/blue values of the bosonic condensate $\rho_{10}(\xi)$ correspond to different phases of the $\mathcal{N}=2^{*}$ plasma. We also show that there is $\xi=\rho^{*}<\rho_{\text {crit }}$ such that for all $\xi>\rho^{*}, \mathcal{N}=2^{*}$ plasma becomes unstable both thermodynamically and dynamically, in agreement with general arguments of [35].

Figs. 2-3 represent $\rho_{10}^{\prime}(\xi), a_{h}^{\prime}(\xi)$, and a numerical verification of the first law of thermodynamics (3.31). Since our numerical data is rather densely spaced ${ }^{17}$ in $\xi$, we

\footnotetext{
${ }^{17}$ Mostly we have $\Delta \xi=10^{-4}$.
} 
use

$$
f^{\prime}(\xi)=\frac{d f(\xi)}{d \xi}=\frac{f(\xi+\Delta \xi)-f(\xi-\Delta \xi)}{2 \Delta \xi},
$$

as a numerical approximation for a derivative of a function $f(\xi)$. Notice that

$$
\lim _{\xi \rightarrow \rho_{\text {crit }}-0} \frac{d \rho_{10}(\xi)}{d \xi}=\mp \infty, \quad \lim _{\xi \rightarrow \rho_{\text {crit }}-0} \frac{d a_{h}(\xi)}{d \xi}=\mp \infty,
$$

with minus/plus signs for red/blue segments of the curves in Fig. 2/Fig. 3 correspondingly. Also, from Fig. 3

$$
\lim _{\xi \rightarrow+0} \frac{d \rho_{10}(\xi)}{d \xi}=+\infty, \quad \lim _{\xi \rightarrow+0} \frac{d a_{h}(\xi)}{d \xi}=+\infty
$$

We find that

$$
\left.\rho_{10}^{\prime}(\xi)\right|_{\xi=+2 \times 10^{-4}} \approx-2.10(6),
$$

which is rather close to a predicted value from the high temperature analysis of section $4(\operatorname{see}(4.7))$

$$
\left.\rho_{10}^{\prime}(\xi)\right|_{\xi=0} ^{\text {high temperature }}=-3 \ln 2 \approx-2.07944 .
$$

A better agreement is achieved if we fit the first 200 points of the red segment of $\rho_{10}(\xi)$ on the left plot in Fig. 1 with a 10th order polynomial in $\xi$. We find in this case

$$
\left.\rho_{10}^{\prime}(\xi)\right|_{\xi=0} ^{\text {fit }} \approx-2.07972
$$

The green points on the right plots in Figs. 2-3 demonstrate the cancellation of $a_{h}^{\prime}(\xi)$ with a combination

$$
4 \xi+2\left(\rho_{10}(\xi)-\xi \frac{d \rho_{10}(\xi)}{d \xi}\right),
$$

as required by the first law of thermodynamics, see (3.31). We find that such a cancellation is achieved with an accuracy of better than $10^{-4}$. Clearly, this provides a rather impressive check on both the validity of the holographic renormalization of the thermal PW flows explained in [24], and the numerical procedures developed in this paper.

\subsection{Supersymmetric deformation}

Albeit more complicated, the Sturm-Liouville problem for the supersymmetric mass deformation is conceptually similar to the one for the bosonic mass deformation studied 

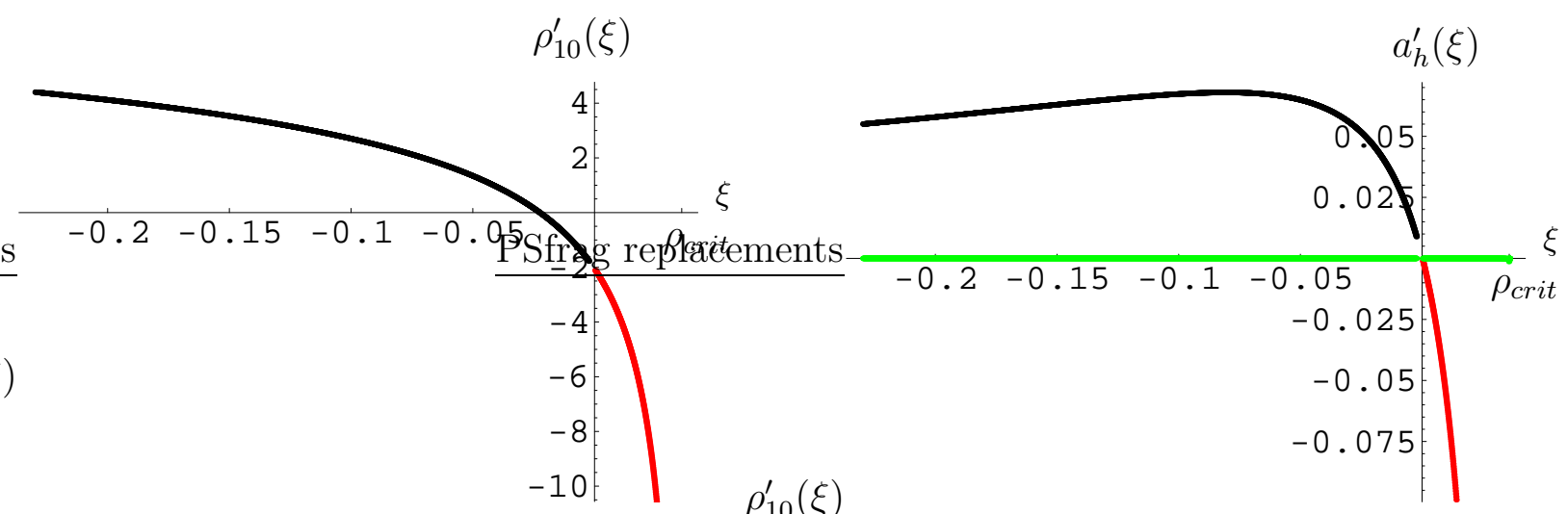

Figure 2: The left plot represents $\rho_{10}^{\prime}(\xi)$ for both the red and the black segments of the left plot in Fig. 1. The right plot represents $a_{h}^{\prime}(\xi)$ for both the red and the black segments of the right plot in Fig. 1. The green points on the right plot represent numerical verification of the first law of the thermodynamics, where $a_{h}^{\prime}(\xi)$ must be canceled with an appropriate combination of $\left\{\xi, \rho_{10}(\xi), \rho^{\prime}(\xi)\right\}$, see $(3.31)$.

in previous subsection. Here we have to use all equations in (2.11). As for the bosonic deformation, we rewrite these equations using $\{\rho(x), a(x)\}$ such that

$$
\alpha \equiv \ln \rho, \quad \ln c_{2}(x) \equiv A(x) \equiv \ln \hat{\delta}_{3}-\frac{1}{4} \ln \left(2 x-x^{2}\right)+a(x) .
$$

The boundary conditions (which guarantee that the flow is singularity-free) are given by $(2.15)$ and $(2.31)$

$$
\begin{array}{llrl}
x \rightarrow 0_{+} & a(x) \rightarrow 0, & \rho(x) \rightarrow 1, & \chi(x) \rightarrow 0 \\
x \rightarrow 1_{-} & a^{\prime}(x) \rightarrow 0, & \rho^{\prime}(x) \rightarrow 0, & \chi^{\prime}(x) \rightarrow 0 .
\end{array}
$$

For the bosonic mass deformation we have a one-dimensional Sturm-Liouville problem: given $\rho_{11}$, the boundary condition at the horizon determines $\rho_{10}$; the pair $\left\{\rho_{11} \equiv\right.$ $\left.\xi, \rho_{10}(\xi)\right\}$ further determines the value of the $a(x)$ at the horizon, $a_{h}(\xi)$. For the supersymmetric mass deformation the Sturm-Liouville problem is two-dimensional: given $\rho_{11} \equiv \xi$, the boundary condition (5.19) would determine

$$
\rho_{10} \equiv \rho_{10}(\xi), \quad \chi_{10} \equiv \chi_{10}(\xi)
$$

For a flow satisfying (5.21) we can extract

$$
a_{h}(\xi)=\lim _{x \rightarrow 1_{-}} a(x)
$$



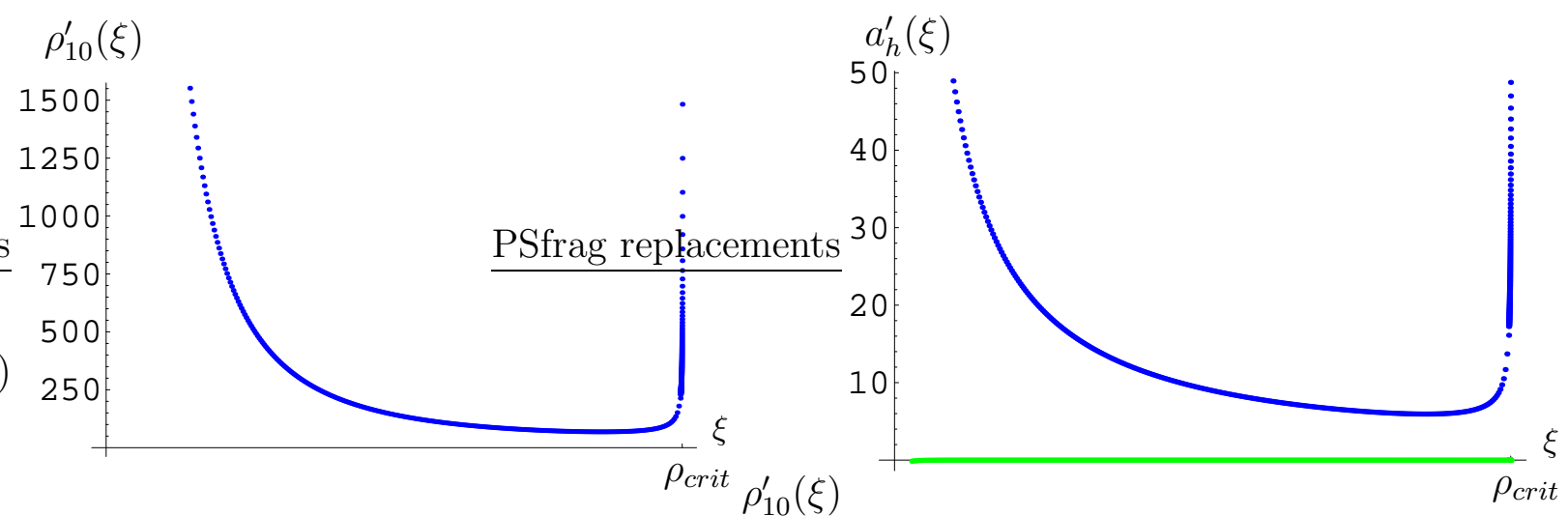

Figure 3: The left plot represents $\rho_{10}^{\prime}(\xi)$ for the blue segment of the left plot in Fig. 1. The right plot represents $a_{h}^{\prime}(\xi)$ for the blue segment of the right plot in Fig. 1 . The green points on the right plot represent numerical verification of the first law of the thermodynamics, where $a_{h}^{\prime}(\xi)$ must be canceled with an appropriate combination of $\left\{\xi, \rho_{10}(\xi), \rho^{\prime}(\xi)\right\}$, see $(3.31)$.

Thus, each non-singular supersymmetric mass deformation flow would generate a set of four numbers

$$
\left\{\xi, \rho_{10}(\xi), \chi_{10}(\xi), a_{h}(\xi)\right\}
$$

which can be used to evaluate the free energy $\mathcal{F}_{\text {susy }}(3.33)$, the speed of sound $c_{s, \text { susy }}^{2}$ (3.27) and (3.34), and verify (numerically) the first law of thermodynamics (3.35).

Since the Sturm-Liouville problem is technically more complex here, we restrict our discussion to $\xi \geq 0$, or following (3.12) $\mathrm{m}^{2} \geq 0$. Results of the numerical analysis are presented in Figs. (4)-(5). Fitting the first 200 points of $\rho_{10}(\xi)$ and $\chi_{10}(\xi)$ with a 10 th order polynomial in $\xi$ we find

$$
\left.\rho_{10}^{\prime}(\xi)\right|_{\xi=0} ^{f i t}=-2.069(2),\left.\quad \chi_{10}\right|_{\xi=0} ^{f i t}=-0.3219(7),
$$

which should be compared with the high temperature predictions ${ }^{18}$ (4.7)

$$
\begin{aligned}
\left.\rho_{10}^{\prime}(\xi)\right|_{\xi=0} ^{\text {high temperature }} & =-3 \ln 2 \approx-2.07944, \\
\left.\chi_{10}(\xi)\right|_{\xi=0} ^{\text {high temperature }} & =-\frac{2^{1 / 2} \Gamma\left(\frac{3}{4}\right)^{4}}{\pi^{2}} \approx-0.3231(1) .
\end{aligned}
$$

\footnotetext{
${ }^{18}$ We reduce the accuracy compare to the bosonic mass deformation case in order to speed-up computations. For a selected set of 41 sets $(5.23)$ we improve accuracy by roughly a factor of $10^{2}$. More accurate data does not provide noticeable quantitative difference.
} 

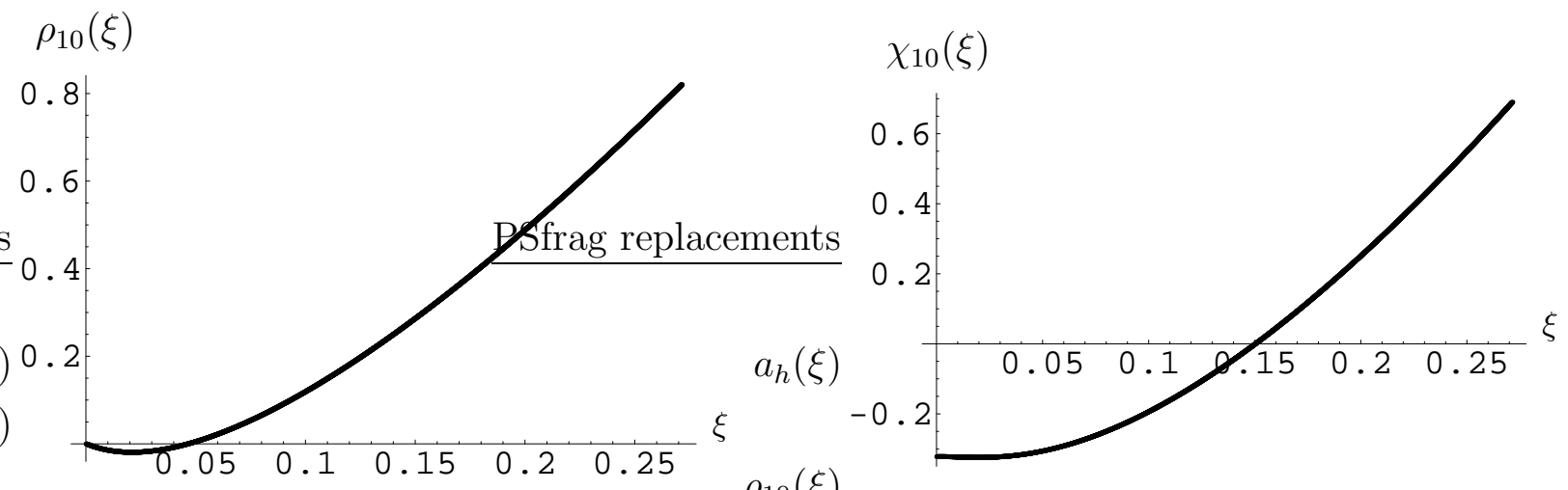

Figure 4: Altogether we obtained 2712 sets $\left\{\xi, \rho_{10}(\xi), \chi(\xi), a_{h}(\xi)\right\}$. The left plot represents $\rho_{10}(\xi)$, and the right plot represents $\chi_{10}(\xi)$.

We do not find a signature for the existence of $\rho_{\text {crit }}$ - it appears for supersymmetric deformation the boundary value problem (5.20) always have a solution ${ }^{19}$.

Fig. 5 represent $a_{h}(\xi)$ (the left plot) and a numerical verification of the first law of thermodynamics (3.35) (the right plot):

$$
\begin{aligned}
\{0\}_{\text {green }}= & \left\{2\left(\rho_{10}(\xi)-\xi \frac{d \rho_{10}(\xi)}{d \xi}\right)\right\}_{\text {blue }}+\left\{-\left(\chi_{10}(\xi)-\xi \frac{d \chi_{10}(\xi)}{d \xi}\right)\right\}_{\text {black }} \\
& +\left\{\frac{d a_{h}(\xi)}{d \xi}\right\}_{\text {red }},
\end{aligned}
$$

where subscripts refer to the color of the points on the right plot in Fig. 5. We find that (5.26) holds with an accuracy of $10^{-3}$.

\subsection{Generic deformation}

Though in this paper we study two special cases of the thermal PW flows - one corresponding to a bosonic mass deformation where the supergravity scalar $\chi(x) \equiv 0$, and the other one corresponding to a supersymmetric mass deformation where the ratio of the coefficients of the non-normalizable modes of the supergravity scalars $\{\rho(x), \chi(x)\}$ is the same as for the supersymmetric PW flow - the numerical methods developed here can be applied for a generic mass deformation as well. In fact, the Sturm-Liouville problem one has to solve in a generic case is exactly the same as in the supersymmetric

\footnotetext{
${ }^{19}$ Recall for a bosonic deformation $\rho_{\text {crit }} \approx-0.0358(2)$ which is well inside the set of $\xi$ considered in Fig. 4.
} 

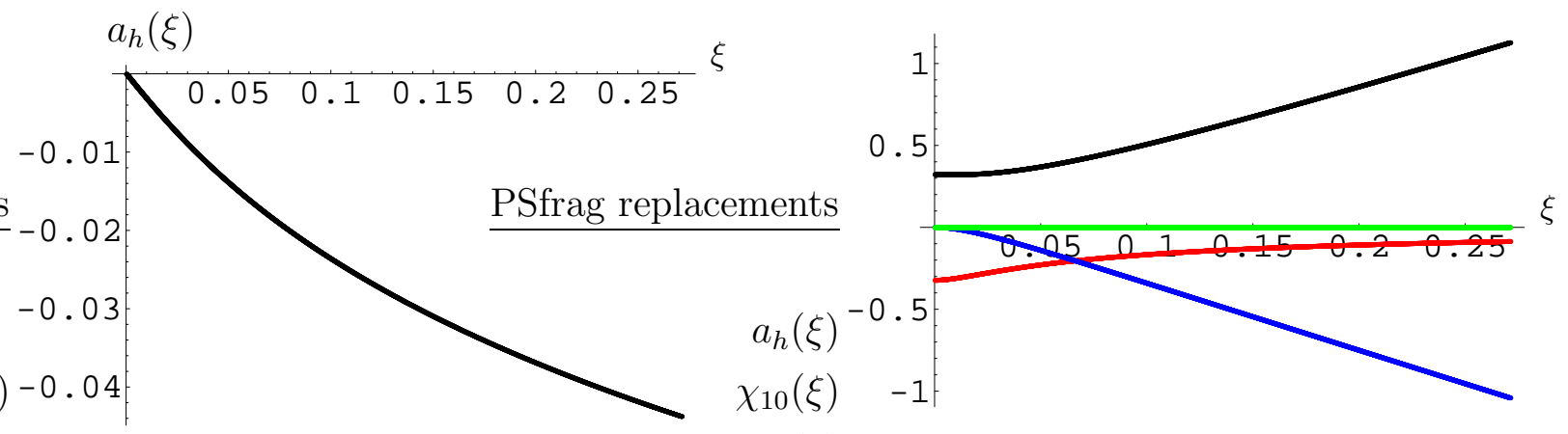

Figure 5: The left plot represents $a_{h}(\xi)$ for the supersymmetric mass deformation. The red/blue/black points on the right plot represent $\alpha_{h}^{\prime}(\xi), 2\left(\rho_{10}-\xi \rho_{10}^{\prime}(\xi)\right)$ and $-\left(\chi_{10}(\xi)-\xi \chi_{10}^{\prime}(\xi)\right)$ correspondingly. The green points on the right plot represent numerical verification of the first law of the thermodynamics, see (3.35).

case where the boundary conditions are given by (5.20). The only difference from the supersymmetric mass deformation case is the (fixed) relation between the coefficients of the non-normalizable modes of the supergravity scalars $\{\rho(x), \chi(x)\}$ given by $(3.14)$

$$
\eta^{2}=6 \frac{m_{f}^{2}}{m_{b}^{2}} \xi .
$$

Recall (see Fig.1) that when $m_{f}=0$ there is a critical value $\rho_{\text {crit }}$, such that for $\xi>\rho_{\text {crit }}$ there is no nonsingular thermal PW flow. On the other hand (see Fig.4), we do not find the evidence for $\rho_{\text {crit }}$ for the supersymmetric mass deformation $m_{f}=m_{b}$. Thus we expect that

$$
\rho_{\text {crit }} \equiv \rho_{\text {crit }}\left(\Delta \equiv \frac{m_{f}^{2}}{m_{b}^{2}}\right),
$$

such that $\rho_{\text {crit }} \rightarrow \infty$ as $\frac{m_{f}^{2}}{m_{b}^{2}} \rightarrow \Delta^{*} \leq 1$. As we show in subsection 6.1 the existence of $\rho_{\text {crit }}$ for $m_{f}=0$ implies destabilization of the thermal plasma at low temperatures. What we appear to be finding is that this particular instability is cured by turning on $m_{f} \neq 0$. Verifying the latter conjecture is beyond the scope of this paper. However, we would like to point out a different type of instability in a system closely related to the one studies here which is eliminated by turning on sufficiently large $m_{f}$. Consider thermal PW flow with the flat $R^{3}$ in (2.7) compactified on a three torus ${ }^{20} T^{3}$. This represents holographic

\footnotetext{
${ }^{20}$ Since a three torus is locally the same as $R^{3}$, such a compactification does not change any of the above computations.
} 

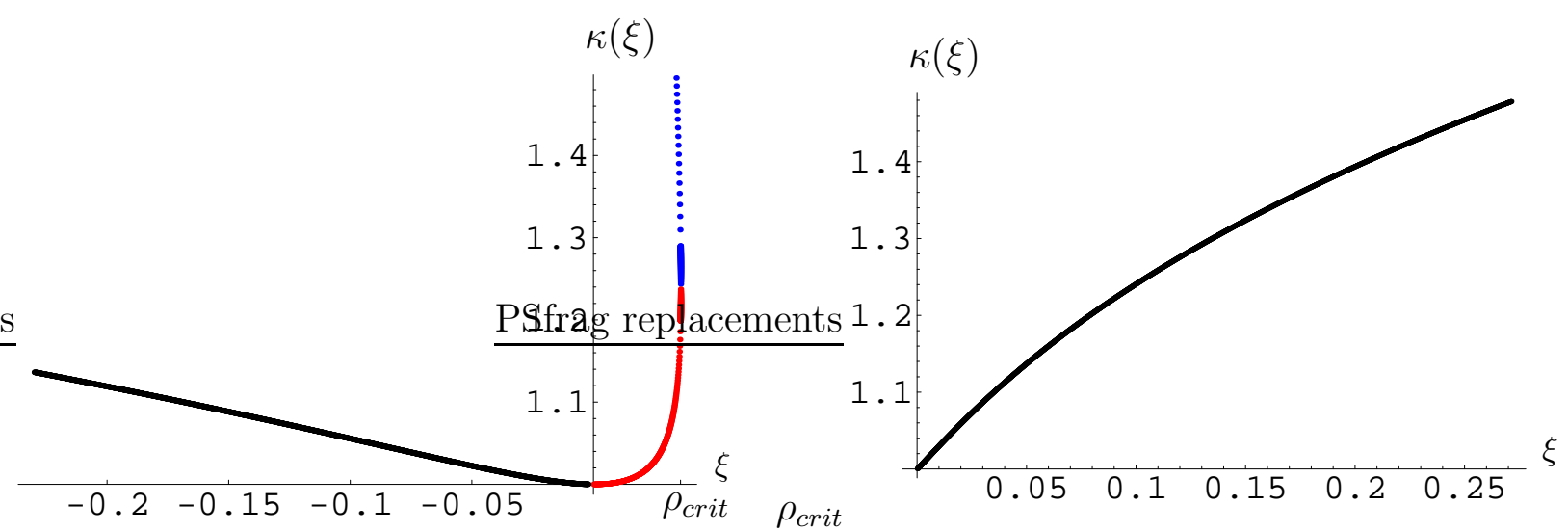

Figure 6: The left plot represents ratio of thermal PW geometry horizon curvature to its curvature at the boundary for bosonic mass deformation. The right plot represents this ratio for the supersymmetric mass deformation.

dual to thermal $\mathcal{N}=2^{*}$ plasma on finite volume $T^{3}$. Analysis identical to that in [38] shows that this system is generically non-perturbatively unstable with respect to a spontaneous creation of the $D 3 \overline{D 3}$ brane pairs ${ }^{21}$. This instability is suppressed provided $^{22}$

$$
\chi_{0}^{2} \geq 6 \rho_{11} \geq 0 \quad \Longleftrightarrow \quad \eta^{2} \geq 6 \xi \geq 0 \quad \Rightarrow \quad \Delta \equiv \frac{m_{f}^{2}}{m_{b}^{2}} \geq 1 .
$$

So, for the nonperturbative instability due to $D 3 \overline{D 3}$ spontaneous brane pair creation for the thermal PW flow compactified on $T^{3}$ we find that $\Delta^{*}=1$.

\subsection{Validity of the supergravity approximation}

Our analysis of the holographic dual to thermal $\mathcal{N}=2^{*}$ gauge theory plasmas are done in the supergravity approximation to type IIb string theory. In the planar limit considered here the string loop corrections are suppressed and the supergravity approximation is valid provided curvature invariants of the thermal PW flow geometry (2.7) are small. We find that along the flow from the boundary to the horizon the absolute value of the Ricci scalar of the five dimensional thermal PW geometry (2.7) increases. As a quantitative criteria for such an increase we propose the ratio of the

\footnotetext{
${ }^{21}$ Similar instability was first identified in [39].

${ }^{22}$ See eq. (4.16) of [38] where one has to remove $(-2)$ from the inequality (coming from the curvature of the 3 -space) and replace $\rho_{11}$ of [38] with $4 \rho_{11}$ due to a different choice of a radial coordinate.
} 
scalar curvature at the horizon to the scalar curvature near the boundary

$$
\kappa \equiv \frac{R^{\text {horizon }}}{R^{\text {boundary }}}=\frac{\mathcal{P}^{\text {horizon }}}{\mathcal{P}^{\text {boundary }}}=-\frac{4}{3} \mathcal{P}^{\text {horizon }}
$$

where we used (2.5) and the fact that $\mathcal{P}^{\text {boundary }}=-\frac{3}{4}$. Values ${ }^{23}$ of $\kappa(\xi)$ for both bosonic and supersymmetric mass deformations are presented in Fig. 6. Notice that for the 'blue' phase

$$
\lim _{\xi \rightarrow 0+} \kappa_{\text {blue }}^{\text {bosonic }}(\xi)=+\infty,
$$

and thus supergravity approximation for the blue phase of the bosonic mass deformation for small values of $\xi$ is not valid.

\section{$6 \mathcal{N}=2^{*}$ plasma at finite temperature}

In this section we discuss thermodynamics of the $\mathcal{N}=2^{*}$ gauge theory plasma for two special cases:

- bosonic deformation, when $m_{f}=0$ and $m_{b} \neq 0$;

- supersymmetric deformation, when $m_{f}=m_{b}=m$.

\subsection{Thermal PW flows with $m_{f}=0, m_{b} \neq 0$}

As explained in subsection 5.1, for the bosonic deformation we observe two phases ('red' phase and 'blue' phase) of the thermal gauge theory plasma for mass deformation parameter $\xi \in\left(0, \rho_{\text {crit }}\right)$ characterized by two different values for the bosonic condensate $\rho_{10}(\xi)$. These are the red and the blue sets of points in Fig. 1. For large values of the bosonic deformation parameter $\left(\xi>\rho_{\text {crit }}\right.$ in Fig. 1$)$ thermal $\mathcal{N}=2^{*}$ gauge theory plasma with bosonic deformation discussed does not exist. The set of black points in Fig. 1 corresponds to $\xi<0$, which following (3.17) corresponds to $m_{b}^{2}<0$. As we argued in subsection 5.4, we can trust supergravity approximation for the red set of points (everywhere) and for black points over the range we studies (corresponding to $\left.m_{b}^{2} / T^{2}<-15\right)$. The supergravity approximation for the blue set of point is much less reliable ${ }^{24}$, especially for small values of $\xi$.

\footnotetext{
${ }^{23}$ In order to evaluate $\mathcal{P}^{\text {horizon }}$ besides $a_{h}(\xi)$ we retained horizon values of the supergravity scalars $\{\rho(x), \chi(x)\}$ while solving the Sturm-Liouville problem (5.4) or (5.20).

${ }^{24}$ The smallest absolute value horizon curvature for the blue points is the same as the largest horizon curvature for the red points.
} 


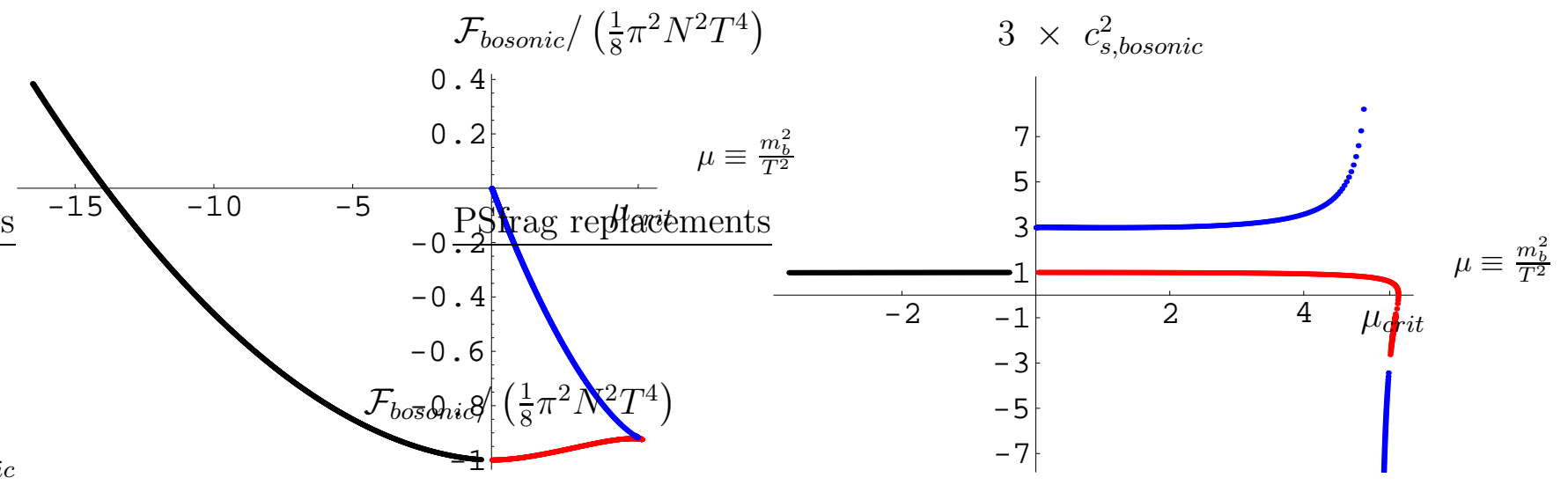

Figure 7: The left plot represents bosonic mass deformation free energy $\mathcal{F}_{\text {bosonic }}$ as a function of $\mu \equiv \frac{m_{b}^{2}}{T^{2}}$. The critical mass is at $\mu_{\text {crit }} \approx 5.28(5)$. The right plot represents speed of sound squared $c_{s, \text { bosonic }}^{2}$ for bosonic mass deformation as a function of $\mu$. Recall that the speed of sound in the conformal plasma $c_{s, C F T}^{2}=\frac{1}{3}$.

We use (3.17), (3.27), (3.29) and (3.30) to evaluate the free energy density and the speed of sound for the bosonic mass deformation. For the free energy density we set an overall (arbitrary) constant part of the free energy ${ }^{25}$

$$
\mathcal{F}_{0}^{\text {bosonic }}=0 \text {. }
$$

Notice that while in the supersymmetric case one can determine $\mathcal{F}_{0}^{\text {susy }}$ unambiguously (see $(3.36))$ by requiring the $\mathcal{F}_{\text {susy }}(T=0)=0$, we can not do so for the bosonic deformation: as we describe shortly, the bosonic deformation becomes unstable both thermodynamically and dynamically at low temperatures. Results of the analysis are presented in Figs. 7-8.

The left plot in Fig. 7 describes the free energy density for the bosonic deformation $\mathcal{F}_{\text {bosonic }}$ relative (up to a sign) to the free energy density of the conformal gauge theory plasma

$$
\mathcal{F}_{C F T}=-\frac{1}{8} \pi^{2} N^{2} T^{4}
$$

as a function of

$$
\mu \equiv \frac{m_{b}^{2}}{T^{2}}
$$

Notice that the blue phase has always a larger (or equal) free energy than the red phase

$$
\mathcal{F}_{\text {bosonic,red }}(\mu) \leq \mathcal{F}_{\text {bosonic,blue }}(\mu)
$$

\footnotetext{
${ }^{25}$ Such ambiguity is present both in thermal quantum field theory and in the framework of the holographic renormalization of the string theory duals to thermal gauge theories.
} 


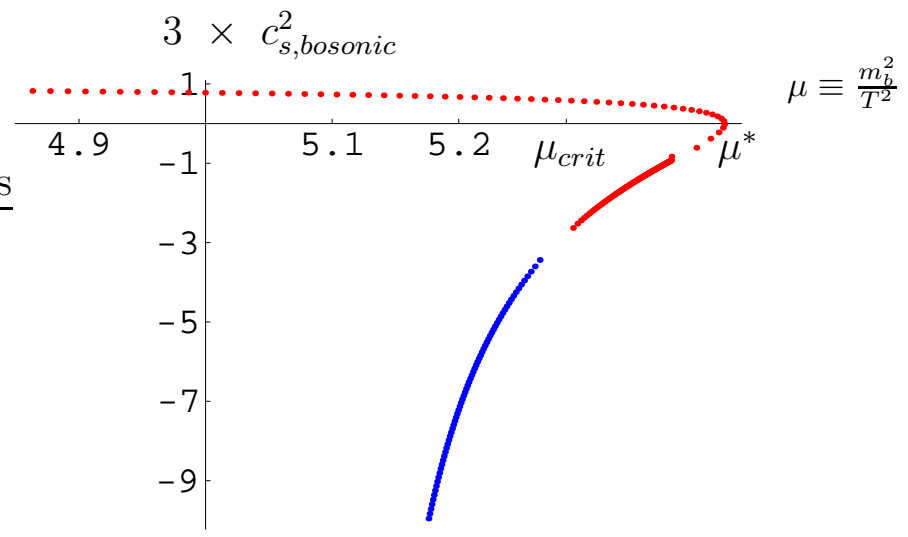

Figure 8: More detailed plot of the speed of sound squared $c_{s, \text { bosonic }}^{2}$ for bosonic mass deformation as a function of $\mu$. The speed of sound vanishes at $\mu^{*} \approx 5.40(9)$. Notice that though $\mu^{*}>\mu_{\text {crit }}$, corresponding to $\mu^{*}$ value of $\xi^{*} \approx 0.035(1)$ is less than $\rho_{\text {crit }} \approx$ $0.03582(2)$, see Fig. 1. Recall that the speed of sound in the conformal plasma $c_{s, C F T}^{2}=$ $\frac{1}{3}$.

with equality achieved only at $\mu=\mu_{\text {crit }}$ corresponding to $\rho_{\text {crit }}$ according to (3.17), (6.3). For the red phase

$$
\frac{\mathcal{F}_{\text {bosonic,red }}(\mu=0)}{\frac{1}{8} \pi^{2} N^{2} T^{4}}=-1,
$$

in agreement with the fact that for $m_{b}=0$ (or at very high temperatures $T \rightarrow \infty$ ) the free energy density must be that of the conformal gauge theory.

The right plot in Fig. 7 and a more detailed plot in Fig. 8 present the speed of sound squared for the thermal $\mathcal{N}=2^{*}$ gauge theory plasma, relative to the speed of sound in the conformal gauge theory plasma

$$
c_{s, C F T}^{2}=\frac{1}{3},
$$

for bosonic deformation as a function of $\mu$. Although bosonic deformation phase discussed here does not exist for $\xi>\rho_{\text {crit }}$, bosonic phase actually extends to values of $\mu_{\text {crit }}<\mu \leq \mu^{*}$ (see Fig. 8). The reason for this is that relation between $\xi$ and $\mu$ as given by (3.17) involves a factor $e^{6 a_{h}(\xi)}$ and is not monotonic around $\xi \sim \rho_{\text {crit }}$. A value of $\xi^{*}$ corresponding to $\mu^{*}$ is actually less than $\rho_{\text {crit }}$ :

$$
\mu^{*} \approx 5.40(9)>\mu_{\text {crit }} \approx 5.28(5) \quad \Rightarrow \quad \xi^{*} \approx 0.035(1)<\rho_{\text {crit }} \approx 0.03582(2) .
$$


We find that the speed of sound for the blue phase is superlunimal, which is yet another indication that this phase is unphysical. The speed of sound diverges for the blue phase as one approach $\mu_{\text {crit }}$ either from above or below. The speed of sound for the red phase at $\mu=0$ is that as in the CFT plasma; it vanishes at $\mu=\mu^{*}$ and becomes purely imaginary for $\xi^{*}<\xi<\rho_{\text {crit }}$ (still for the red phase and well within supergravity approximation)

$$
\begin{aligned}
& c_{s, \text { bosonic,red }}^{2}(\mu=0)=\frac{1}{3}, \quad c_{s, \text { bosonic,red }}^{2}\left(\mu=\mu^{*}\right)=0, \\
& c_{s, \text { bosonic,red }}^{2}\left(\mu \equiv 12 \sqrt{2} \pi^{2} e^{6 a_{h}(\xi)} \xi\right)<0, \quad \xi^{*}<\xi<\rho_{\text {crit }} .
\end{aligned}
$$

We would like to stress that conclusions (6.8) are very robust and in fact, immediately follow from the existence of $\rho_{\text {crit }}$ for the bosonic condensate $\rho_{10}(\xi)$. Indeed, existence of $\rho_{\text {crit }}$, i.e., impossibility to determine $\rho_{10}(\xi)$ for $\xi>\rho_{\text {crit }}$, along with the assumption for the smoothness of $\rho_{10}(\xi)$, implies that $\rho_{10}(\xi)$ is not a single valued function of $\xi$ around $\rho_{\text {crit }}$; moreover,

$$
\lim _{\xi \rightarrow \rho_{\text {crit }-0}} \rho_{10}^{\prime}(\xi)=\mp \infty,
$$

with the minus sign in the red phase and the plus sign in the blue phase (precisely what is presented in Fig. 1). But then from (3.30)

$$
\lim _{\xi \rightarrow \rho_{\text {crit }}-0} \Sigma_{\text {bosonic }}(\xi)=\mp \infty,
$$

again with the minus sign in the red phase and the plus sign in the blue phase. Thus using (3.27) we see that in the red phase

$$
\lim _{\xi \rightarrow \rho_{\text {crit }}-0}\left(3 \times c_{s, \text { bosonic }}^{2}(\xi)\right)=-3,
$$

(in agreement with Figs. 7-8), and for the blue phase

$$
\lim _{\xi \rightarrow \xi_{\infty} \mp 0}\left(3 \times c_{s, \text { bosonic }}^{2}(\xi)\right)= \pm \infty, \quad \sum_{\text {bosonic }, \text { blue }}\left(\xi_{\infty}\right)=3
$$

where $0<\xi_{\infty}<\rho_{\text {crit }}$. Since in the red phase at $\xi=0$ the speed of sound squared is $\frac{1}{3}>$ 0 and given (6.11) there is a guaranteed value of $\xi^{*}<\rho_{\text {crit }}$ at which the speed of sound vanishes, and for $\xi^{*}<\xi<\rho_{\text {crit }}$ the speed of sound is purely imaginary. As emphasized in [35], a thermal system with $c_{s}^{2}<0$ is unstable simultaneously thermodynamically (it has a negative specific heat) and dynamically (amplitude of small pressure/energy density fluctuations exponentially grows). 

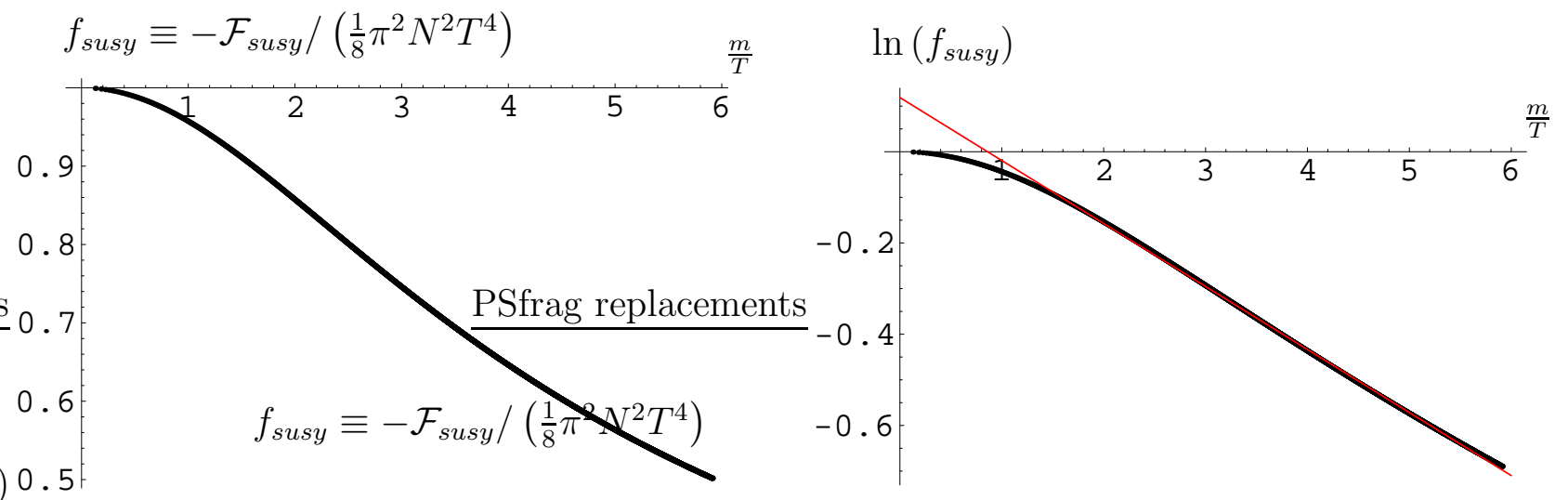

Figure 9: The left plot represents supersymmetric mass deformation free energy $\mathcal{F}_{\text {susy }}$ as a function of $\frac{m}{T}$. The right plot represents $\ln \left(f_{\text {susy }}\right)$ as a function of $\frac{m}{T}$ (black points) and a straight line fit (red curve) for the last 2500 points of the distribution.

Finally, we do not see thermodynamic instability in thermal $\mathcal{N}=2^{*}$ plasma with bosonic mass deformation for $m_{b}^{2}<0$, see Fig. 7. In fact, we find that for negative values of $\mu$ the speed of sound slowly (and monotonically) decreases - it reaches about $90 \%$ of the conformal plasma speed of sound (6.6) at ${ }^{26} \mu \approx-35$. Of course, this does not exclude the fact that thermal $\mathcal{N}=2^{*}$ plasma with $m_{b}^{2}<0$ might have dynamical instability without accompanying thermodynamics instability [35].

\subsection{Thermal PW flows with $m_{f}=m_{b} \equiv m$}

Bosonic and fermionic condensates $\left\{\rho_{10}(\xi), \chi_{10}(\xi)\right\}$ for thermal $\mathcal{N}=2^{*}$ gauge theory plasma with supersymmetric mass deformation are presented in Fig. 4. As we argued in subsection 5.4, supergravity approximation here is valid of the whole range of deformation parameter $\xi$ considered. We use (3.17), (3.27), (3.33), (3.34) and (3.36) to evaluate the free energy density and the speed of sound for the supersymmetric mass deformations. Results of the analysis are presented in Figs. 9-10. We do not see any signature of the phase transition ${ }^{27}$, or the thermodynamic instability.

Probably the most unexpected result of the analysis is that strongly coupled thermal $\mathcal{N}=2^{*}$ plasma for supersymmetric mass deformation behaves much closer to a conformal plasma than one would naively expect. The right plot on Fig. 9 presents the logarithm of the ratio of the $\mathcal{N}=2^{*}$ plasma free energy density to the free energy

\footnotetext{
${ }^{26}$ This is outside the range of the right plot in Fig. 7 .

${ }^{27}$ This would show up as the free energy $\mathcal{F}_{\text {susy }}\left(\frac{m}{T}\right)$ changing sign for a particular value of $\frac{m}{T}$.
} 


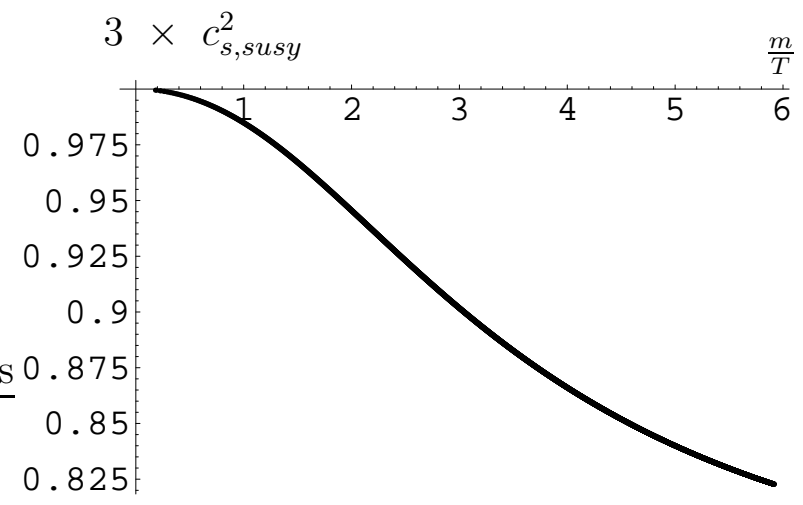

Figure 10: The speed of sound squared $c_{s, \text { susy }}^{2}$ for supersymmetric mass deformation as a function of $\frac{m}{T}$.

density of the conformal plasma (6.2)

$$
\ln \left(f_{\text {susy }}\right) \equiv \ln \frac{\mathcal{F}_{\text {susy }}}{\mathcal{F}_{C F T}},
$$

as a function of $\frac{m}{T}$. We did a straight line line fit (solid red line on the right plot on Fig. 9) for the low temperature ${ }^{28}$

$$
\left.\ln \left(f_{\text {susy }}\right)\right|^{F I T}(x)=0.118(8)-0.138(1) x .
$$

Thus, to a good approximation the free energy of the thermal $\mathcal{N}=2^{*}$ plasma can be approximated by

$$
\mathcal{F}_{\text {susy }}^{\text {approx }} \approx 1.1(3) \times \mathcal{F}_{C F T} e^{-\frac{m}{7.2(4) T}} \propto-T^{4} e^{-\frac{m}{7 T}},
$$

so that noticeable deviation from conformality occurs at $T \sim \frac{1}{7} m$.

It is instructing to compare (6.15) with the zero coupling result for the $\mathcal{N}=2^{*}$ plasma. At zero coupling we have ${ }^{29} N^{2}$ massless $\mathcal{N}=2$ vector multiples and $N^{2}$ massive $\mathcal{N}=2$ hypermultiplets. The free energy density of the massless states is just $\frac{2}{3} \mathcal{F}_{C F T}$ in (6.2), while the free energy density of a mass $m$ spin $s$ particle is given by $[40,41]$

$$
\mathcal{F}^{s}=-\frac{(2 s+1) m^{2} T^{2}}{2 \pi^{2}} \sum_{\ell=1}^{\infty} \frac{\eta^{\ell+1}}{\ell^{2}} K_{2}\left(\frac{\ell m}{T}\right)
$$

\footnotetext{
${ }^{28}$ In practice, we define low temperatures as the subset of the mass deformation parameters $\frac{m}{T}>\sim 1$.

${ }^{29}$ We neglect $(-1)$ in the large $N$ limit.
} 
where $\eta= \pm 1$ for bosons/fermions correspondingly. As low temperatures $\frac{m}{T} \gg 1$ we can use asymptotic expansion for the Bessel function $K_{2}(x)$

$$
K_{2}(x) \approx \sqrt{\frac{\pi}{2 x}} e^{-x}, \quad x \gg 1
$$

to conclude that

$$
\mathcal{F}^{s} \approx-\frac{(2 s+1) m^{3 / 2} T^{5 / 2}}{(2 \pi)^{3 / 2}} e^{-\frac{m}{T}}, \quad \frac{m}{T} \gg 1
$$

Altogether we find that the free energy of the thermal $\mathcal{N}=2^{*}$ plasma for supersymmetric mass deformation at zero coupling is

$$
\mathcal{F}_{\mathcal{N}=2^{*}}=-\frac{\pi^{2} N^{2} T^{4}}{12}\left(1+\frac{24 \sqrt{2}}{\pi^{7 / 2}}\left(\frac{m}{T}\right)^{3 / 2} e^{-\frac{m}{T}}\right), \quad \frac{m}{T} \gg 1
$$

From (6.19), we find that the free energy density is roughly $60 \%$ of the high temperature result at $T \sim m$, while the corresponding number for the strongly coupled plasma (6.14) is about $98 \%$.

The hydrodynamic properties and the jet quenching of strongly coupled nonconformal gauge theory plasma are typically parameterized in terms of $[25,42] \delta$

$$
\delta \equiv\left(\frac{1}{3}-c_{s}^{2}\right)
$$

For the strongly coupled $\mathcal{N}=2^{*}$ thermal plasma at ${ }^{30} T \sim 1.5 m$ we find (see Fig. 10)

$$
\left.\delta^{\mathcal{N}=2^{*}}\right|_{T \approx 1.5 m} \approx 2 \times 10^{-3}
$$

which further emphasizes the striking similarity between strongly coupled $\mathcal{N}=2^{*}$ plasma and conformal $\mathcal{N}=4$ gauge theory plasma.

\section{Conclusion and future directions}

In this paper we presented detailed analysis of the thermodynamics of the strongly coupled $\mathcal{N}=2^{*}$ gauge theory plasma. We considered two special cases of the mass deformations: bosonic mass deformation (where only the bosonic components of the

\footnotetext{
${ }^{30}$ The temperature of the quark-gluon plasma at RHIC is of order the QCD deconfinement temperature [43].
} 

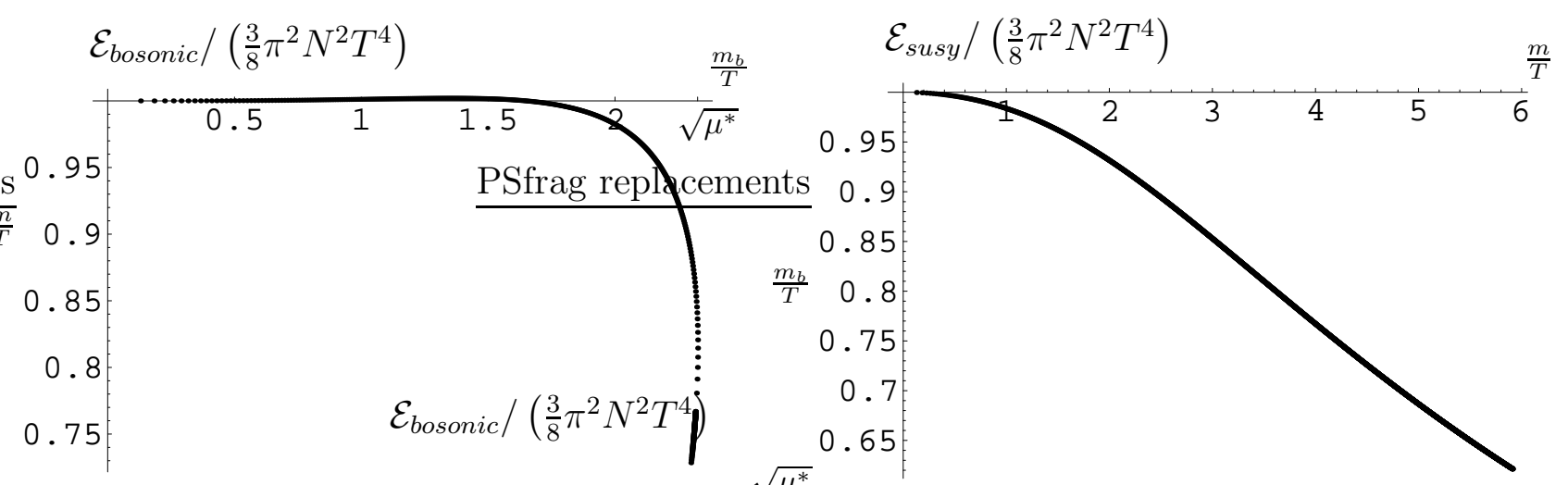

Figure 11: The left plot represents bosonic mass deformation energy density $\mathcal{E}_{\text {bosonic }}$ as a function of $\frac{m_{b}}{T}$. The value $\sqrt{\mu^{*}}$ (see Fig. 8) denotes the ratio $\frac{m_{b}}{T}$ for which the speed of sound vanishes. The right plot represents supersymmetric mass deformation energy density $\mathcal{E}_{\text {susy }}$ as a function of $\frac{m}{T}$.

$\mathcal{N}=2$ hypermultiplet get nonzero mass) and supersymmetric mass deformation (with bosonic and fermionic components of the hypermultiplet getting the same mass). We argued that both our theoretical framework and the numerical procedures are under control, given a highly nontrivial consistency check of the first law of thermodynamics for the extracted data. We also argued that the supergravity approximation is under control, as the curvature invariants of the dual geometry remain small.

The most important result of the analysis is the striking similarity between thermodynamics of the mass deformed $\mathcal{N}=2^{*}$ gauge theory plasma at temperatures of order the mass scale and the thermodynamics of the conformal $\mathcal{N}=4 \mathrm{SYM}$ plasma. We would like to reemphasize the latter fact by presenting the plots for the energy energy for various mass deformations, see Fig. 11.

We could like to concluded with some open problems.

- In is important to generalize our analysis to other examples of the strongly coupled nonconformal gauge theory plasma. Most notably the cascading gauge theory plasma [18-20, 22].

- It is interesting to evaluate hydrodynamic properties and jet quenching in $\mathcal{N}=2^{*}$ gauge theory plasma in the low temperature regime.

- The speed of sound waves in $\mathcal{N}=2^{*}$ gauge theory plasma for the bosonic mass deformation was found to vanish for certain value of $\frac{m_{b}}{T}$. It is interesting to study hydrodynamics of the gauge theory plasma in the regime of the vanishing speed of 
sound and determine the critical exponent of the bulk viscosity.

\section{Acknowledgments}

We would like to thank Jaume Gomis, Chris Herzog, Pavel Kovtun, Gerry McKeon and Andrei Starinets for interesting discussions. We would like to thank Ofer Aharony and Rob Myers for valuable comments on the manuscript. AB's research at Perimeter Institute is supported in part by the Government of Canada through NSERC and by the Province of Ontario through MEDT. AB gratefully acknowledges further support by an NSERC Discovery grant. JTL's research was supported in part by the US Department of Energy under grant DE-FG02-95ER40899 and the National Science Foundation under grant PHY99-07949. JTL acknowledges the hospitality of the KITP, where this work was initiated.

\section{References}

[1] J. M. Maldacena, "The large N limit of superconformal field theories and supergravity," Adv. Theor. Math. Phys. 2, 231 (1998) [Int. J. Theor. Phys. 38, 1113 (1999)] [arXiv:hep-th/9711200].

[2] O. Aharony, S. S. Gubser, J. M. Maldacena, H. Ooguri and Y. Oz, "Large N field theories, string theory and gravity," Phys. Rept. 323, 183 (2000) [arXiv:hepth/9905111].

[3] H. Liu, K. Rajagopal and U. A. Wiedemann, "Wilson loops in heavy ion collisions and their calculation in AdS/CFT," arXiv:hep-ph/0612168.

[4] K. Adcox et al. [PHENIX Collaboration], "Formation of dense partonic matter in relativistic nucleus nucleus collisions at RHIC: Experimental evaluation by the PHENIX collaboration," Nucl. Phys. A 757, 184 (2005) [arXiv:nucl-ex/0410003].

[5] B. B. Back et al., "The PHOBOS perspective on discoveries at RHIC," Nucl. Phys. A 757, 28 (2005) [arXiv:nucl-ex/0410022].

[6] I. Arsene et al. [BRAHMS Collaboration], "Quark gluon plasma and color glass condensate at RHIC? The perspective from the BRAHMS experiment," Nucl. Phys. A 757, 1 (2005) [arXiv:nucl-ex/0410020]. 
[7] J. Adams et al. [STAR Collaboration], "Experimental and theoretical challenges in the search for the quark gluon plasma: The STAR collaboration's critical assessment of the evidence from RHIC collisions," Nucl. Phys. A 757, 102 (2005) [arXiv:nucl-ex/0501009].

[8] S. S. Gubser, I. R. Klebanov and A. W. Peet, Phys. Rev. D 54, 3915 (1996) [arXiv:hep-th/9602135].

[9] S. S. Gubser, I. R. Klebanov and A. A. Tseytlin, "Coupling constant dependence in the thermodynamics of $\mathrm{N}=4$ supersymmetric Yang-Mills theory," Nucl. Phys. B 534, 202 (1998) [arXiv:hep-th/9805156].

[10] G. Policastro, D. T. Son and A. O. Starinets, "The shear viscosity of strongly coupled N = 4 supersymmetric Yang-Mills plasma," Phys. Rev. Lett. 87, 081601 (2001) [arXiv:hep-th/0104066].

[11] A. Buchel, J. T. Liu and A. O. Starinets, "Coupling constant dependence of the shear viscosity in $\mathrm{N}=4$ supersymmetric Yang-Mills theory," Nucl. Phys. B 707, 56 (2005) [arXiv:hep-th/0406264].

[12] P. Benincasa and A. Buchel, "Transport properties of $\mathrm{N}=4$ supersymmetric YangMills theory at finite coupling," JHEP 0601, 103 (2006) [arXiv:hep-th/0510041].

[13] H. Liu, K. Rajagopal and U. A. Wiedemann, "Calculating the jet quenching parameter from AdS/CFT," Phys. Rev. Lett. 97, 182301 (2006) [arXiv:hepph/0605178].

[14] C. P. Herzog, A. Karch, P. Kovtun, C. Kozcaz and L. G. Yaffe, JHEP 0607, 013 (2006) [arXiv:hep-th/0605158].

[15] H. Liu, K. Rajagopal and U. A. Wiedemann, "An AdS/CFT calculation of screening in a hot wind," arXiv:hep-ph/0607062.

[16] S. Caron-Huot, P. Kovtun, G. D. Moore, A. Starinets and L. G. Yaffe, "Photon and dilepton production in supersymmetric Yang-Mills plasma," arXiv:hepth/0607237.

[17] E. V. Shuryak, "Strongly coupled quark-gluon plasma: The status report," arXiv:hep-ph/0608177. 
[18] A. Buchel, "Finite temperature resolution of the Klebanov-Tseytlin singularity," Nucl. Phys. B 600, 219 (2001) [arXiv:hep-th/0011146].

[19] A. Buchel, C. P. Herzog, I. R. Klebanov, L. A. Pando Zayas and A. A. Tseytlin, "Non-extremal gravity duals for fractional D3-branes on the conifold," JHEP 0104, 033 (2001) [arXiv:hep-th/0102105].

[20] S. S. Gubser, C. P. Herzog, I. R. Klebanov and A. A. Tseytlin, "Restoration of chiral symmetry: A supergravity perspective," JHEP 0105, 028 (2001) [arXiv:hepth/0102172].

[21] A. Buchel and J. T. Liu, "Thermodynamics of the $\mathrm{N}=2^{*}$ flow," JHEP 0311, 031 (2003) [arXiv:hep-th/0305064].

[22] O. Aharony, A. Buchel and A. Yarom, "Holographic renormalization of cascading gauge theories," Phys. Rev. D 72, 066003 (2005) [arXiv:hep-th/0506002].

[23] D. Mateos, R. C. Myers and R. M. Thomson, "Thermodynamics of the brane," arXiv:hep-th/0701132.

[24] A. Buchel, "N = 2* hydrodynamics," Nucl. Phys. B 708, 451 (2005) [arXiv:hepth/0406200].

[25] P. Benincasa, A. Buchel and A. O. Starinets, "Sound waves in strongly coupled non-conformal gauge theory plasma," Nucl. Phys. B 733, 160 (2006) [arXiv:hepth/0507026].

[26] A. Buchel, A. W. Peet and J. Polchinski, "Gauge dual and noncommutative extension of an $\mathrm{N}=2$ supergravity solution," Phys. Rev. D 63, 044009 (2001) [arXiv:hep-th/0008076].

[27] K. Pilch and N. P. Warner, "N = 2 supersymmetric RG flows and the IIB dilaton," Nucl. Phys. B 594, 209 (2001) [arXiv:hep-th/0004063].

[28] R. Donagi and E. Witten, "Supersymmetric Yang-Mills Theory And Integrable Systems," Nucl. Phys. B 460, 299 (1996) [arXiv:hep-th/9510101].

[29] J. Polchinski and M. J. Strassler, "The string dual of a confining four-dimensional gauge theory," arXiv:hep-th/0003136. 
[30] A. Buchel and J. T. Liu, "Universality of the shear viscosity in supergravity," Phys. Rev. Lett. 93, 090602 (2004) [arXiv:hep-th/0311175].

[31] N. J. Evans, C. V. Johnson and M. Petrini, "The enhancon and $\mathrm{N}=2$ gauge theory/gravity RG flows," JHEP 0010, 022 (2000) [arXiv:hep-th/0008081].

[32] A. Buchel, "Comments on fractional instantons in $\mathrm{N}=2$ gauge theories," Phys. Lett. B 514, 417 (2001) [arXiv:hep-th/0101056].

[33] S. S. Gubser, I. R. Klebanov and A. W. Peet, "Entropy and Temperature of Black 3-Branes," Phys. Rev. D 54, 3915 (1996) [arXiv:hep-th/9602135].

[34] S. S. Gubser and I. Mitra, "Instability of charged black holes in anti-de Sitter space," arXiv:hep-th/0009126.

[35] A. Buchel, "A holographic perspective on Gubser-Mitra conjecture," Nucl. Phys. B 731, 109 (2005) [arXiv:hep-th/0507275].

[36] J. O. Andersen, E. Petitgirard and M. Strickland, "Two-loop HTL thermodynamics with quarks," Phys. Rev. D 70, 045001 (2004) [arXiv:hep-ph/0302069].

[37] A. Buchel, "Higher derivative corrections to near-extremal black holes in type IIB supergravity," Nucl. Phys. B 750, 45 (2006) [arXiv:hep-th/0604167].

[38] A. Buchel, "Gauge theories on hyperbolic spaces and dual wormhole instabilities," Phys. Rev. D 70, 066004 (2004) [arXiv:hep-th/0402174].

[39] J. M. Maldacena and L. Maoz, "Wormholes in AdS," JHEP 0402, 053 (2004) [arXiv:hep-th/0401024].

[40] J. I. Kapusta and C. Gale, "Finite-Temperature Field Theory: Principles and Applications," Cambridge Monographs on Mathematical Physics (2006).

[41] A. O. Starinets, "Effective potentials in quantum field theory at finite temperature", Cand. Sci. Dissertation (1994), in Russian.

[42] A. Buchel, "On jet quenching parameters in strongly coupled non-conformal gauge theories," Phys. Rev. D 74, 046006 (2006) [arXiv:hep-th/0605178].

[43] E. V. Shuryak, "What RHIC experiments and theory tell us about properties of quark-gluon plasma?," Nucl. Phys. A 750, 64 (2005) [arXiv:hep-ph/0405066]. 